This article is published with open access at Springerlink.com

\title{
A construction of cylindric and polyadic algebras from atomic relation algebras
}

\section{IAN HODKINSON}

\begin{abstract}
Given a simple atomic relation algebra $\mathcal{A}$ and a finite $n \geq 3$, we construct effectively an atomic $n$-dimensional polyadic equality-type algebra $\mathcal{P}$ such that for any subsignature $L$ of the signature of $\mathcal{P}$ that contains the boolean operations and cylindrifications, the $L$-reduct of $\mathcal{P}$ is completely representable if and only if $\mathcal{A}$ is completely representable. If $\mathcal{A}$ is finite then so is $\mathcal{P}$.

It follows that there is no algorithm to determine whether a finite $n$-dimensional cylindric algebra, diagonal-free cylindric algebra, polyadic algebra, or polyadic equality algebra is representable (for diagonal-free algebras this was known). We also obtain a new proof that the classes of completely representable $n$-dimensional algebras of these types are non-elementary, a result that remains true for infinite dimensions if the diagonals are present, and also for infinite-dimensional diagonal-free cylindric algebras.
\end{abstract}

\section{Introduction}

Algebraic logic has traditionally studied representations of various kinds of abstract algebra as genuine algebras of relations on a set. A representation is an embedding from the abstract algebra into a concrete algebra of relations, respecting the operations on the algebra. One example is relation algebras. These are axiomatically-defined abstract algebras whose corresponding concrete algebras are algebras of binary relations on some base set, the concrete algebra operations being the boolean operations, identity (or equality), relational converse, and the relational composition of two binary relations. Another example is $n$-dimensional cylindric algebras, for some fixed ordinal $n$. Again, these are abstract algebras, defined by axioms; the corresponding concrete algebras are algebras of $n$-ary relations on some base set, endowed with the boolean operations together with diagonals and cylindrifications, which are algebraic versions of first-order equality and existential quantification, respectively. In these algebras, in finite dimensions, all non-permutational substitution operations are definable — these operations are algebraic analogues of changing the free variables in a first-order formula. Polyadic equality algebras are similar to cylindric algebras, but include all substitutions as primitive

Presented by J. Raftery.

Received August 24, 2011; accepted in final form February 22, 2012.

2010 Mathematics Subject Classification: Primary: 03G15.

Key words and phrases: algebraic logic, algebras of relations, cylindric algebra, diagonalfree cylindric algebra, polyadic algebra, polyadic equality algebra, complete representation, undecidable, non-elementary.

The research was partially supported by UK EPSRC grant GR/S19905/01. 


\begin{tabular}{|c|c|c|c|c|}
\hline & diagonals & cylindrifications & sub & stitutions \\
\hline algebras & & & all & non-1-1 \\
\hline substitution algebras & & & & $\checkmark$ \\
\hline $\begin{array}{c}\text { diagonal-free cylindric } \\
\text { algebras }\end{array}$ & & $\checkmark$ & & \\
\hline cylindric algebras & $\checkmark$ & $\checkmark$ & & definable \\
\hline polyadic algebras & & $\checkmark$ & $\checkmark$ & \\
\hline polyadic equality algebras & $\checkmark$ & $\checkmark$ & $\checkmark$ & \\
\hline
\end{tabular}

TABLE 1. Some algebras of finite-dimensional relations.

operations. If we drop the diagonals, we obtain polyadic algebras, and if we drop the substitutions as well, we obtain diagonal-free cylindric algebras. Substitution algebras (Pinter, [24, Definition 2.1]) incorporate only the boolean operations and non-permutational substitutions. See Table 1 for a summary.

An abstract algebra is said to be representable if it has a representation. Often, the class of abstract representable algebras is difficult to characterise. For example, it is frequently not finitely axiomatisable in first-order logic (e.g., $[21,22])$. Rather less studied than questions of axiomatisation is the question of whether an algorithm exists to ascertain representability of finite abstract algebras. There is a connection between the two kinds of question. If the class of representable algebras is finitely axiomatisable (in almost any finitary logic one can think of), then such an algorithm exists: we simply evaluate the finite set of axioms in the given algebra. (Thus, for example, by [5, 3.2.54, $3.2 .55,3.2 .65]$, for $n \leq 2$ it is decidable whether a finite $n$-dimensional cylindric algebra is representable.) But the converse is false in general, and the class $\mathrm{Crs}_{n}$ (for finite $n \geq 3$ ) of $n$-dimensional cylindric relativised set algebras provides a counter-example: see [23] and [5, 5.5.12]. So showing that there is no algorithm to decide representability gives a stronger result than non-finite axiomatisability of the class of representable algebras in a particular logic.

In $[7,8]$, it was shown that the problem of whether a finite relation algebra is representable is indeed undecidable. The proof involved a rather intricate reduction of an undecidable tiling problem. Some applications appear in $[10$, 11]. The result was extended in [10] to finite $n$-dimensional diagonal-free cylindric algebras, for all finite $n \geq 3$, using work of Johnson [12].

A little can be deduced from this about the cylindric algebra case. In [20], Monk gave an effective representability-preserving construction of a 3dimensional cylindric algebra from an arbitrary relation algebra. [20, p. 63] states that the idea is due to Lyndon. [20, p. 81] adds that 'This description occurs in a letter from Lyndon to Thompson dated May, 1949 . . in this letter he restricts himself to the case of proper relation algebras.' It also states that reference to the embedding has occurred in several places. The earliest of them 
is [15]. Now if the relation algebra is finite, then the constructed cylindric algebra will also be finite. It follows by Turing reduction from the relation algebra case that it is undecidable whether a finite 3-dimensional cylindric algebra is representable.

It has remained an open question whether there is an algorithm to decide representability of finite cylindric algebras of higher finite dimensions $(4,5, \ldots)$. That there is no such algorithm can be proved by a complicated adaptation of the already rather complicated proof for relation algebras, and this has been done in outline by Robin Hirsch and independently by the author. However, it would be simpler, perhaps more interesting, and potentially more useful, to generalise Monk's construction, at least for finite or atomic relation algebras, to higher dimensions.

In some way, this has already been done. In [16], Maddux put forward a new construction of cylindric algebras from relation algebras, using what we will call 'networks'. Here is a rough summary adequate for now (we will say more in Section 3). In dimension 3, given a relation algebra, Maddux's construction produces a 3-dimensional cylindric algebra isomorphic to Monk's. The construction can also work in higher dimensions. In dimension 4, given a relation algebra, it produces a 4-dimensional cylindric algebra, but representability may not be preserved. In higher dimensions, it works only for certain relation algebras and again representability may not be preserved.

In this paper, we present a construction that (roughly) does preserve and reflect representability in higher dimensions than 3. Our construction is similar to Maddux's in the case of finite algebras and dimension 3.

In a little more detail, let $\mathcal{A}$ be any simple atomic relation algebra. (We assume simplicity solely to allow a shorter presentation, and simple algebras are all we need for our undecidability result — by [8, Theorem 18.13], it is undecidable whether a finite simple relation algebra is representable.) Let $n \geq 3$ be finite. We will construct from $\mathcal{A}$ an atomic $n$-dimensional polyadic equality-type algebra $\mathcal{P}_{n}(\mathcal{A})$ with the following property. Let $L$ be any subsignature of the signature of polyadic equality algebras containing the boolean operators and cylindrifications — that is, $L$ lies between diagonal-free cylindric algebras and polyadic equality algebras in expressivity. Let $\mathcal{Q}$ be the $L$-reduct of $\mathcal{P}_{n}(\mathcal{A})$. Then $\mathcal{A}$ has a complete representation if and only if $\mathcal{Q}$ has a complete representation. See Theorem 4.13 below; a rough explanation of the construction will be given in Section 3. (A complete representation is one that respects all existing meets and joins in the algebra.) If $\mathcal{A}$ is finite, the construction is effective and $\mathcal{Q}$ is finite, and in this case there is of course no difference between complete representations and ordinary representations. It follows by Turing reduction from the relation algebra case [7, 8] that for any finite $n \geq 3$, there is no algorithm to decide whether a finite L-algebra is representable as an algebra of relations. See Corollary 5.1 below.

This theorem covers cylindric algebras, diagonal-free cylindric algebras, polyadic algebras, and polyadic equality algebras (Theorem 5.2), and the proof 
handles all of them together. It does not cover, e.g., Pinter's substitution algebras, whose signature is just the booleans and the non-permutational substitution operators - and indeed their representability is decidable since the class of representable algebras is finitely axiomatisable [25, Theorem 17].

Our construction has further applications. In [6], it was shown that the classes of completely representable relation algebras, and completely representable $\alpha$-dimensional cylindric algebras, for any $\alpha \geq 3$, are non-elementary. Here we prove that for any finite $n \geq 3$ and any signature $L$ as above, the class of completely representable $L$-algebras is non-elementary, again by 'reduction' from the relation algebra case. See Corollary 6.3. Essentially the same result can be found in [13], which adapts the known proof for cylindric algebras. Remark 6.4 discusses the infinite-dimensional case: when diagonals are present, the direct argument used for cylindric algebras in [6] can be applied, and we can also handle infinite-dimensional diagonal-free cylindric algebras as a special case. As far as we know, the case of infinite-dimensional polyadic algebras remains open.

Layout of paper. After revision of some background information in Section 2, the construction is presented in Section 3, the proof of preservation of representability in Section 4, the undecidability result in Section 5, and the nonelementary result in Section 6. We hope that the construction will find further uses and be generalised to other kinds of algebra, and Section 7 lists some possibilities.

Notation. We will generally identify (notationally) an algebra or structure with its domain. For sets $X, Y, \wp(X)$ denotes the power set of $X$, and ${ }^{X} Y$ denotes the set of maps $f: X \rightarrow Y$. Maps are regarded formally as sets of ordered pairs, so above, $f=\{(x, f(x)): x \in X\}$. For a partial map $f: X \rightarrow Y$, we write $\operatorname{dom} f$ for the domain $\{x: \exists y((x, y) \in f)\}$ of $f$. For possibly partial maps $f: X \rightarrow Y$ and $g: Y \rightarrow Z$, we let $g \circ f$ denote the composition map $: X \rightarrow Z$ given by $g \circ f(x)=g(f(x)$ ) (for $x \in \operatorname{dom} f$ with $f(x) \in \operatorname{dom} g$ ). For $f: X \rightarrow Y$ and $X^{\prime} \subseteq X$, we write $f\left[X^{\prime}\right]$ for $\left\{f(x): x \in X^{\prime}\right\}$, and $\operatorname{rng} f$ for $f[X]$. For $f, g: X \rightarrow Y$ and $x \in X$, we write $f={ }_{x} g$ if $f\left(x^{\prime}\right)=g\left(x^{\prime}\right)$ for each $x^{\prime} \in X \backslash\{x\}$. An ordinal is the set of all smaller ordinals. For an ordinal $n$, we generally write elements of ${ }^{n} X$ as $\bar{a}, \bar{b}$ (to suggest $n$-tuples or sequences), and write $\bar{a}(i)$ simply as $a_{i}$ for $i<n$.

\section{Background}

We assume familiarity with the fundamentals of boolean algebras, some experience with basic aspects of relation algebras ([5, 5.3.1-5.3.5] and [18] have more than what we need), and a little acquaintance with cylindric algebras and polyadic algebras. In this section, we briefly recall some relevant notions and fix some notation. Fix, throughout, a finite dimension $n \geq 3$ (so $n=$ $\{0,1, \ldots, n-1\})$. 
2.1. Boolean algebras. Let $\mathcal{A}, \mathcal{B}$ be similar algebras of a signature containing the boolean operators, and suppose that the boolean reducts of $\mathcal{A}, \mathcal{B}$ are boolean algebras. As usual, by an atom of $\mathcal{A}$ we will mean an atom of its boolean reduct. We write At $\mathcal{A}$ for the set of atoms of $\mathcal{A}$. A homomorphism $f: \mathcal{A} \rightarrow \mathcal{B}$ is said to be complete if $f\left(\sum^{\mathcal{A}} S\right)=\sum^{\mathcal{B}} f[S]$ whenever $S \subseteq \mathcal{A}$ and $\sum^{\mathcal{A}} S$ exists (equivalently, $f\left(\prod^{\mathcal{A}} S\right)=\prod^{\mathcal{B}} f[S]$ whenever $S \subseteq \mathcal{A}$ and $\prod^{\mathcal{A}} S$ exists). The composition of two complete homomorphisms is complete. We will need the following lemma, which is essentially [8, 2.16-2.17].

Lemma 2.1. Let $\mathcal{A}, \mathcal{B}$ be as above and assume that $\mathcal{B}$ is atomic. Let $f: \mathcal{A} \rightarrow \mathcal{B}$ be a homomorphism. Then $f$ is complete iff for each atom $b \in$ At $\mathcal{B}$, there is an atom $a \in \operatorname{At} \mathcal{A}$ with $f(a) \geq b$. If $f$ is complete and injective, then $\mathcal{A}$ is atomic.

Proof. If $f$ is complete, take $b \in \operatorname{At} \mathcal{B}$. The set $S=\{a \in \mathcal{A}: f(a) \geq b\}$ is an ultrafilter of $\mathcal{A}$, so $a=\prod^{\mathcal{A}} S$ exists (it is an atom of $\mathcal{A}$ in $S$ if $S$ is principal, and 0 otherwise). If $a=0$, then as $f$ is complete, $0=f(a)=\prod^{\mathcal{B}} f[S] \geq b>0$, a contradiction. So $a \in S$ is an atom of $\mathcal{A}$ with $f(a) \geq b$.

Conversely, assume the condition and take any $x \in \mathcal{A}$ and $S \subseteq \mathcal{A}$ such that $x=\sum^{\mathcal{A}} S$. Then $f(x)$ is an upper bound in $\mathcal{B}$ for $f[S]$. Assume for contradiction that $f(x) \neq \sum^{\mathcal{B}} f[S]$. As $\mathcal{B}$ is atomic, there is $b \in$ At $\mathcal{B}$ with $b \leq f(x)$ and $b \cdot f(s)=0$ for all $s \in S$. Using the condition, take $a \in$ At $\mathcal{A}$ with $f(a) \geq b$. Then $f(a \cdot x)=f(a) \cdot f(x) \geq b>0$, so $a \cdot x>0$ and $a \leq x$. Hence, $a \leq s$ for some $s \in S$ (else $x-a$ is a smaller upper bound for $S$ ). So $b \leq f(a) \leq f(s)$, contradiction.

For the last part, if $x \in \mathcal{A}$ is non-zero, then $f(x)>0$, so as $\mathcal{B}$ is atomic, there is $b \in \operatorname{At} \mathcal{B}$ with $b \leq f(x)$. Take $a \in \operatorname{At} \mathcal{A}$ with $f(a) \geq b$. As above, $a \leq x$, proving that $\mathcal{A}$ is atomic.

2.2. Representations and complete representations. A relation algebra is an algebra $\mathcal{A}=\left(A,+,-, 0,1,1^{\prime},{ }^{\smile}, ;\right)$ satisfying certain equations laid down in $[26]$ (see also $[5,5.3 .1],[19,6.0 .1]$ ). The equations comprise the axioms for boolean algebras (so $(A,+,-, 0,1)$ will be a boolean algebra) plus others that will not be explicitly needed here. See, e.g., $[19,8]$ for more information about relation algebras.

Definition 2.2. A full relation set algebra is an algebra of the form

$$
\mathfrak{R e}(U)=\left(\wp(U \times U), \cup, \backslash, \emptyset, U \times U, I d_{U},-^{-1}, \mid\right),
$$

where $U$ is a set, $I d_{U}=\{(x, x): x \in U\}$, and for $R, S \subseteq U \times U$, we have $R^{-1}=\{(x, y):(y, x) \in R\}$ and $R \mid S=\{(x, y): \exists z((x, z) \in R \wedge(z, y) \in S)\}$.

Let $\mathcal{A}$ be a relation algebra. A representation of $\mathcal{A}$ is an embedding (i.e., an injective homomorphism) $h: \mathcal{A} \rightarrow \mathcal{R}=\prod_{k \in K} \mathfrak{R e}\left(U_{k}\right)$, for some index set $K$ and sets $U_{k}(k \in K)$. We say that $h$ is an atomic representation if for every $k \in K$ and $x, y \in U_{k}$, there is an atom $a$ of $\mathcal{A}$ with $(x, y) \in h(a)$, and is a complete representation if $h: \mathcal{A} \rightarrow \mathcal{R}$ is a complete homomorphism in the 
sense of Subsection 2.1. We say that $\mathcal{A}$ is (completely) representable if it has at least one (complete) representation.

This does indeed 'represent' the elements of $\mathcal{A}$ as relations because, assuming that the $U_{k}$ are pairwise disjoint, $h(a)$ (for $a \in \mathcal{A}$ ) can be identified with the binary relation $\bigcup_{k \in K} h(a)_{k}$ on $\bigcup_{k \in K} U_{k}$. There are similar definitions for polyadic equality algebras and their reducts. An ( $n$-dimensional) polyadic equality-type algebra is an algebra with the signature

$$
\left\{+,-, 0,1, \mathrm{~d}_{i j}, \mathrm{c}_{i}, \mathrm{~s}_{\sigma}: i, j<n, \sigma: n \rightarrow n\right\},
$$

where the boolean operations $+,-, 0,1$ are as for relation algebras, the $\mathrm{d}_{i j}$ are constants, and the $c_{i}$ and $\mathbf{s}_{\sigma}$ are unary function symbols. In this paper, we do not need to restrict ourselves to polyadic equality algebras (algebras satisfying the axioms of polyadic equality algebra), so we do not introduce the polyadic axioms. (See, e.g., [5, §5.4] for more information.)

Definition 2.3. An (n-dimensional) full polyadic equality set algebra is an $n$-dimensional polyadic equality-type algebra of the form

$$
\mathfrak{P e}_{n}(U)=\left(\wp\left({ }^{n} U\right), \cup, \backslash, \emptyset,{ }^{n} U, D_{i j}^{U}, C_{i}^{U}, S_{\sigma}^{U}: i, j<n, \sigma: n \rightarrow n\right),
$$

where $U$ is a set, and for each $X \subseteq{ }^{n} U, i, j<n$, and $\sigma: n \rightarrow n$,

- $D_{i j}^{U}=\left\{\bar{a} \in{ }^{n} U: a_{i}=a_{j}\right\}$,

- $C_{i}^{U} X=\left\{\bar{a} \in{ }^{n} U: \exists \bar{b} \in X\left(\bar{a}={ }_{i} \bar{b}\right)\right\}$, where $\bar{a}={ }_{i} \bar{b}$ means that $a_{j}=b_{j}$ for each $j \in n \backslash\{i\}$ (as in the Notation in Section 1),

- $S_{\sigma}^{U} X=\left\{\bar{a} \in{ }^{n} U: \bar{a} \circ \sigma \in X\right\}$.

Definition 2.4. Let $\mathcal{B}$ be an $L$-algebra, where

$$
\{+,-, 0,1\} \subseteq L \subseteq\left\{+,-, 0,1, \mathrm{~d}_{i j}, \mathrm{c}_{i}, \mathrm{~s}_{\sigma}: i, j<n, \sigma: n \rightarrow n\right\} .
$$

(1) Suppose that $L \cap\left\{\mathrm{d}_{i j}, \mathrm{~s}_{\sigma}: i, j<n, \sigma: n \rightarrow n\right\} \neq \emptyset$. A representation of $\mathcal{B}$ is an $L$-embedding $h: \mathcal{B} \rightarrow \mathcal{Q}=\prod_{k \in K} \mathcal{Q}_{k}$, where $K$ is some set and each $\mathcal{Q}_{k}$ is the $L$-reduct of a full polyadic equality set algebra $\mathfrak{P} \mathfrak{e}_{n}\left(U_{k}\right)$, for some set $U_{k}$. For use below, we write $P_{k}={ }^{n} U_{k}$ for each $k \in K$.

(2) Suppose instead that $L \subseteq\left\{+,-, 0,1, \mathrm{c}_{i}: i<n\right\}$. In this case, a representation of $\mathcal{B}$ is an $L$-embedding $h: \mathcal{B} \rightarrow \mathcal{Q}=\prod_{k \in K} \mathcal{Q}_{k}$, where $K$ is some set, $U_{k, i}$ is a set (for each $k \in K, i<n$ ), $P_{k}=\prod_{i<n} U_{k, i}$ (for each $k \in K)$, and

$$
\mathcal{Q}_{k}=\left(\wp\left(P_{k}\right), \cup, \backslash, \emptyset, P_{k}, C_{i}^{P_{k}}: i<n, \mathrm{c}_{i} \in L\right),
$$

where $C_{i}^{P_{k}} X=\left\{\bar{a} \in P_{k}: \exists \bar{b} \in X\left(\bar{a}={ }_{i} \bar{b}\right)\right\}$ for each $X \subseteq P_{k}$ and $i<n$.

In either case, if such an $h$ exists, the boolean reduct of $\mathcal{B}$ is a boolean algebra. We say that $h$ is an atomic representation if for every $k \in K$ and $\bar{a} \in P_{k}$, there is an atom $b$ of $\mathcal{B}$ with $\bar{a} \in h(b)$, and is a complete representation if $h$ is complete in the sense of Subsection 2.1. It can be checked that $h$ is complete iff the projection $\pi_{k} \circ h: \mathcal{B} \rightarrow \mathcal{Q}_{k}$ is a complete homomorphism for each $k \in K$. $\mathcal{B}$ is said to be (completely) representable if it has some (complete) representation. 
Remark 2.5. The following applies to all kinds of representation defined so far. Any representation of a finite algebra is complete - all existing meets and joins are finite and so are respected by any representation, since it preserves the boolean operations. The algebras $\mathcal{R}, \mathcal{Q}$ in Definitions 2.2 and 2.4 are atomic, so by Lemma 2.1, a representation of an arbitrary algebra is complete iff it is atomic, and any completely representable algebra is atomic.

If $\mathcal{A}$ is a dense subalgebra of $\mathcal{B}$ - that is, for each $b \in \mathcal{B} \backslash\{0\}$ there is $a \in \mathcal{A} \backslash\{0\}$ with $a \leq b$ - then for each $S \subseteq \mathcal{A}$ such that $\sum^{\mathcal{A}} S$ exists, we have $\sum^{\mathcal{A}} S=\sum^{\mathcal{B}} S$. Hence, if $\mathcal{B}$ is completely representable, then so is any dense subalgebra of $\mathcal{B}$.

An algebra is simple if it has no proper nontrivial homomorphic images. (Some authors also require that the algebra is itself nontrivial.) If $\mathcal{A}$ is a simple relation algebra and $h: \mathcal{A} \rightarrow \mathcal{R}=\prod_{k \in K} \mathfrak{R e}\left(U_{k}\right)$ is a representation, let $\pi_{k}: \mathcal{R} \rightarrow \mathfrak{R e}\left(U_{k}\right)$ be the natural projection for each $k$. It is easily verified that $\pi_{k}$ is a complete homomorphism. As $h$ is one-one, there is $k \in K$ such that $\pi_{k} \circ h(0) \neq \pi_{k} \circ h(1)$. By simplicity, $\pi_{k} \circ h: \mathcal{A} \rightarrow \mathfrak{R e}\left(U_{k}\right)$ is one-one and so a representation of $\mathcal{A}$, and if $h$ is a complete representation, then so is $\pi_{k} \circ h$. Similar considerations apply to the representations of Definition 2.4. We conclude that any simple (completely) representable algebra of the above kinds has a (complete) representation of the above form with $|K|=1$. Such a representation is sometimes said to be square.

2.3. Polyadic equality atom structures. Our algebra $\mathcal{P}_{n}(\mathcal{A})$ will be the complex algebra over an atom structure.

Definition 2.6. An ( $n$-dimensional) polyadic equality atom structure is a structure of the form

$$
\mathcal{S}=\left(S, \mathrm{~d}_{i j}, \equiv_{i},{ }^{\sigma}: i, j<n, \sigma: n \rightarrow n\right),
$$

where $S$ is a non-empty set, $\mathrm{d}_{i j} \subseteq S, \equiv_{i}$ is an equivalence relation on $S$, $-^{\sigma}: S \rightarrow S$ is a map taking $s \in S$ to an element $s^{\sigma} \in S$, and $\left(s^{\sigma}\right)^{\tau}=s^{\sigma \circ \tau}$ for all $\sigma, \tau: n \rightarrow n$ and $s \in S$. We will often refer to elements of an atom structure as 'atoms'.

The complex algebra of $\mathcal{S}$ is the $n$-dimensional polyadic equality-type algebra

$$
\mathcal{S}^{+}=\left(\wp(S), \cup, \backslash, \emptyset, S, \mathrm{~d}_{i j}, \mathrm{c}_{i}, \mathrm{~s}_{\sigma}: i, j<n, \sigma: n \rightarrow n\right),
$$

where for each $X \subseteq S$, we have $c_{i} X=\left\{s \in S: \exists x \in X\left(x \equiv_{i} s\right)\right\}$ and $\mathrm{s}_{\sigma} X=\left\{s \in S: s^{\sigma} \in X\right\}$. As is usual, we identify an element $s \in S$ with the atom $\{s\} \in \mathcal{S}^{+}$.

2.4. Relation algebra networks. The atoms of the atom structure will be made from atomic $\mathcal{A}$-networks. 
Definition 2.7. Let $\mathcal{A}$ be an atomic relation algebra. An atomic $\mathcal{A}$-network is a structure $N=\left(N_{1}, N_{2}\right)$, where $N_{1}$ is a (possibly empty) set of 'nodes', and $N_{2}: N_{1} \times N_{1} \rightarrow$ At $\mathcal{A}$ is a map satisfying, for all $x, y, z \in N_{1}$,

(1) $N_{2}(x, x) \leq 1$

(2) $N_{2}(x, y)=N_{2}(y, x)^{\smile}$,

(3) $N_{2}(x, y) \leq N_{2}(x, z) ; N_{2}(z, y)$.

$N$ is said to be strict if $N_{2}(x, y) \leq 1^{\prime} \Rightarrow x=y$. For networks $N=\left(N_{1}, N_{2}\right)$ and $N^{\prime}=\left(N_{1}^{\prime}, N_{2}^{\prime}\right)$, and an object $i$, we write $N={ }_{i} N^{\prime}$ if $N_{1} \backslash\{i\}=N_{1}^{\prime} \backslash\{i\}$ and $N_{2}(j, k)=N_{2}^{\prime}(j, k)$ for all $j, k \in N_{1} \backslash\{i\}$. Note that we require neither $N_{1}=N_{1}^{\prime}$ nor $i \in N_{1} \cup N_{1}^{\prime}$ here. We sometimes drop indices and just write $N$ for any of $N, N_{1}, N_{2}$. We may refer to $N_{1}$ as the domain of $N$, or its set of nodes.

2.5. Equivalence relations on $n$. Each atom will also incorporate an equivalence relation on $n$ that will determine which diagonals the atom lies in. Here, we lay down some useful facts and notation about such equivalence relations. The number $n-3$ figures prominently and its role will become clear in Section 3 .

Definition 2.8. We write $E q(n)$ for the set of equivalence relations on $n$. Let $\sim, \sim^{\prime} \in E q(n)$.

(1) For $i<n$, we write $\sim={ }_{i} \sim^{\prime}$ iff $j \sim k \Longleftrightarrow j \sim^{\prime} k$ for all $j, k \in n \backslash\{i\}$.

(2) For $X \subseteq n$, we write $X / \sim=\{\{j<n: j \sim i\}: i \in X\}$ (this is slightly non-standard, since we may have $\bigcup(X / \sim) \neq X)$.

(3) We let

$$
\begin{aligned}
H(\sim) & =\{X \subseteq n: n \backslash X \text { is a union of exactly } n-3 \sim \text {-classes }\} \\
& =\{X \subseteq n:|(n \backslash X) / \sim|=n-3 \text { and } \bigcup(X / \sim)=X\} .
\end{aligned}
$$

Of course, $H(\sim)$ may be empty. Obviously, $|X| \leq 3$ for each $X \in H(\sim)$.

(4) For $X, Y \subseteq n$ and $i<n$, we say that $X$ and $Y$ match for $\sim$ off $i$ if $X \cup\{i\}=Y \cup\{i\}=I$, say, and $|(n \backslash I) / \sim|=n-3$.

(5) For $\sigma: n \rightarrow n$, we write $\sim_{\sigma} \in E q(n)$ for the equivalence relation given by $i \sim \sim_{\sigma} j$ iff $\sigma(i) \sim \sigma(j)$ (for each $i, j<n$ ).

(6) For $X \subseteq n$ and a map $\sigma: n \rightarrow n$, we let

$$
\sigma_{\sim}(X)=\{i<n: \forall j<n(i \sim \sigma(j) \rightarrow j \in X)\} .
$$

A more explicit but less handy equivalent definition is

$$
\sigma_{\sim}(X)=n \backslash \bigcup((\sigma[n \backslash X]) / \sim) .
$$

We will need the following elementary lemma. We let Id denote the identity map on $n$.

Lemma 2.9. Let $i<n$, let $\sim, \sim^{\prime} \in E q(n)$ satisfy $\sim={ }_{i} \sim^{\prime}$, and let $X, X^{\prime} \subseteq n$.

(1) If $X$ and $X^{\prime}$ match for $\sim$ off $i$, then $X \backslash\{i\}=X^{\prime} \backslash\{i\}$.

(2) $X$ and $X^{\prime}$ match for $\sim$ off $i$ iff they match for $\sim^{\prime}$ off $i$. 
(3) If $X \in H(\sim)$, then $X$ and $X^{\prime}$ match for $\sim$ off $i$ iff $X \cup\{i\}=X^{\prime} \cup\{i\}=I$, say, and $\operatorname{Id}_{\sim}(I)=X$.

(4) If $\sigma, \tau: n \rightarrow n$ and $\sigma(j) \sim \tau(j)$ for each $j \in n \backslash X$, then $\sigma_{\sim}(X)=\tau_{\sim}(X)$.

(5) If $\sigma: n \rightarrow n$ and $X \in H\left(\sim_{\sigma}\right)$, then

(a) $\sigma[X] \subseteq \sigma_{\sim}(X)$,

(b) $\sigma[n \backslash X] \subseteq n \backslash \sigma_{\sim}(X)$ and $(\sigma[n \backslash X]) / \sim=\left(n \backslash \sigma_{\sim}(X)\right) / \sim$,

(c) $\sigma_{\sim}(X) \in H(\sim)$.

(6) If $\sigma, \tau: n \rightarrow n$, then $(\sigma \circ \tau)_{\sim}(X)=\sigma_{\sim}\left(\tau_{\sim_{\sigma}}(X)\right)$.

Proof. In the proof, we use $j, k, t, u$, and (after part (3)) $i$ as variables ranging over $n$.

(1) and (2): These are easy.

(3): Assume that $X \cup\{i\}=X^{\prime} \cup\{i\}=I$, say. By definition,

$$
\operatorname{Id}_{\sim}(I)=\{t: \forall u(t \sim u \rightarrow u \in I)\} .
$$

Claim. $|(n \backslash I) / \sim|=n-3$ iff $\operatorname{Id}_{\sim}(I) \subseteq X$.

Proof of claim. Plainly, $(n \backslash I) / \sim \subseteq(n \backslash X) / \sim$, and the latter has size $n-3$ since $X \in H(\sim)$. So $|(n \backslash I) / \sim|=n-3$ iff they are equal, iff $(n \backslash I) / \sim \supseteq(n \backslash X) / \sim$, iff $\forall t(t \notin X \rightarrow \exists u(t \sim u \wedge u \notin I))$, iff $\forall t(\forall u(t \sim u \rightarrow u \in I) \rightarrow t \in X)$, which by (2.1), is iff $\operatorname{Id}_{\sim}(I) \subseteq X$. This proves the claim.

Since $X$ is a union of $\sim$-classes and $X \subseteq I$, it is immediate from (2.1) that $X \subseteq \operatorname{Id}_{\sim}(I)$. By the claim, $X$ and $X^{\prime}$ match for $\sim$ off $i$ iff $|(n \backslash I) / \sim|=n-3$, iff $\operatorname{Id}_{\sim}(I)=X$. What we have proved is equivalent to what is required.

(4): Given the assumption, observe simply that $\sigma_{\sim}(X)=\{i: \forall j(j \notin X \rightarrow$ $i \not \sigma(j))\}=\{i: \forall j(j \notin X \rightarrow i \nsim \tau(j))\}=\tau_{\sim}(X)$.

(5): Let $i$ be arbitrary. If $i \in X$, then for any $j$, if $\sigma(i) \sim \sigma(j)$, then $i \sim_{\sigma} j$; as $X$ is a union of $\sim_{\sigma}$-classes, this implies $j \in X$. So by definition, $\sigma(i) \in \sigma_{\sim}(X)$, and we conclude that $\sigma[X] \subseteq \sigma_{\sim}(X)$. If on the other hand $i \notin X$, then $j=i$ satisfies $\sigma(i) \sim \sigma(j)$ and $j \notin X$, so $\sigma(i) \notin \sigma_{\sim}(X)$. Hence, $\sigma[n \backslash X] \subseteq n \backslash \sigma_{\sim}(X)$, so also, $(\sigma[n \backslash X]) / \sim \subseteq\left(n \backslash \sigma_{\sim}(X)\right) / \sim$. Further, if $k \notin \sigma_{\sim}(X)$, then by definition there is $j \notin X$ with $k \sim \sigma(j)$. Hence, $(\sigma[n \backslash X]) / \sim \supseteq\left(n \backslash \sigma_{\sim}(X)\right) / \sim$ as well. It now follows that $\sigma_{\sim}(X) \in H(\sim)$, because it is immediate from the definition that $\sigma_{\sim}(X)$ is a union of $\sim$-classes, and $\left|\left(n \backslash \sigma_{\sim}(X)\right) / \sim\right|=|(\sigma[n \backslash X]) / \sim|=\left|(n \backslash X) / \sim_{\sigma}\right|=n-3$, since $X \in H\left(\sim_{\sigma}\right)$.

(6): We note that for each $i$,

$$
\begin{aligned}
i \in \sigma_{\sim}\left(\tau_{\sim_{\sigma}}(X)\right) & \Longleftrightarrow \forall j\left(i \sim \sigma(j) \rightarrow j \in \tau_{\sim_{\sigma}}(X)\right) \\
& \Longleftrightarrow \forall j\left(i \sim \sigma(j) \rightarrow \forall k\left(j \sim_{\sigma} \tau(k) \rightarrow k \in X\right)\right) \\
& \Longleftrightarrow \forall j(i \sim \sigma(j) \rightarrow \forall k(\sigma(j) \sim \sigma(\tau(k)) \rightarrow k \in X)) \\
& \Longleftrightarrow \forall j k(i \sim \sigma(j) \wedge \sigma(j) \sim \sigma(\tau(k)) \rightarrow k \in X) \\
& \Longleftrightarrow \forall k(\exists j[i \sim \sigma(j) \wedge \sigma(j) \sim \sigma(\tau(k))] \rightarrow k \in X) \\
& \Longleftrightarrow \forall k(i \sim \sigma(\tau(k)) \rightarrow k \in X) \Longleftrightarrow i \in(\sigma \circ \tau)_{\sim}(X) .
\end{aligned}
$$


(Near the end, it is clear that $\exists j[i \sim \sigma(j) \wedge \sigma(j) \sim \sigma(\tau(k))]$ implies that $i \sim \sigma(\tau(k))$ as $\sim$ is an equivalence relation, and the converse follows by taking $j=\tau(k)$.)

\section{The construction}

We begin this section by attempting to outline our construction informally. The reader may wish to skip this description.

In [20], Monk constructed from an arbitrary relation algebra $\mathcal{A}$ a certain 3 -dimensional cylindric algebra, which we will denote here by $\mathcal{C}(\mathcal{A})$. The connection between relation algebras and 3-dimensional cylindric algebras has been explored much further by Maddux — see, e.g., [16, 18] — and in the former, Maddux gave a new construction of cylindric algebras from relation algebras that gives a result isomorphic to $\operatorname{Monk}$ 's $\mathcal{C}(\mathcal{A})$ in dimension 3 . According to this construction, in the case when $\mathcal{A}$ is finite, $\mathcal{C}(\mathcal{A})$ is isomorphic to the complex algebra over the following atom structure $\mathcal{S}(\mathcal{A})$. The elements of $\mathcal{S}(\mathcal{A})$ are all ' 3 -dimensional' atomic $\mathcal{A}$-networks of the form $N=\left(3, N_{2}\right)$ - that is, with set of nodes $\{0,1,2\}$. The diagonal $\mathrm{d}_{i j}$ is $\left\{N \in \mathcal{S}(\mathcal{A}): N(i, j) \leq 1^{\prime}\right\}$, and $N \equiv{ }_{i} N^{\prime}$ iff $N(j, k)=N^{\prime}(j, k)$ for all $j, k \in 3 \backslash\{i\}$. For cylindric algebras, we do not need to introduce the substitutions $-^{\sigma}$ for $\sigma: 3 \rightarrow 3$, though it would be easy to do so by defining $N^{\sigma}$ by $N^{\sigma}(i, j)=N(\sigma(i), \sigma(j))$. Representability of (finite) $\mathcal{A}$ is preserved and reflected by $\mathcal{C}(\mathcal{A})$, very roughly because

(i) a representation of $\mathcal{A}$ can be 'read off' from a representation of $\mathcal{C}(\mathcal{A})$ in a straightforward way using the so-called relation algebra reduct of $\mathcal{C}(\mathcal{A})$, which is isomorphic to $\mathcal{A}$ (see, e.g., [5, 5.3.7] for information on relation algebra reducts), and

(ii) all 3-dimensional atomic $\mathcal{A}$-networks 'embed into' any representation of $\mathcal{A}$ in a way respecting the operations of $\mathcal{C}(\mathcal{A})$, and thus a representation of $\mathcal{C}(\mathcal{A})$ can be interpreted in one of $\mathcal{A}$.

Maddux's construction extends in some way to higher dimensions. For $n \geq 4$, Maddux ([17, Theorem 10]; see also [18] and [5, 5.3.17]) constructed an $n$-dimensional cylindric algebra from any atomic relation algebra $\mathcal{A}$ that has an $n$-dimensional cylindric basis (which is a set of $n$-dimensional atomic $\mathcal{A}$-networks with certain substitution and amalgamation properties). But even for $n=4$, the cylindric algebra may not inherit the representability of $\mathcal{A}$ (see [17, pp. 960-961] and [18, p. 389] for an example), and for $n \geq 5$, not all atomic relation algebras (even representable ones) have an $n$-dimensional cylindric basis. So although this is an important construction, used by a number of authors, it is not what we need here.

Here, we will construct an atomic $n$-dimensional polyadic equality-type algebra $\mathcal{P}_{n}(\mathcal{A})$ from an arbitrary (simple) atomic relation algebra $\mathcal{A}$. To help explain our approach, let us say that a loose representation of $\mathcal{A}$ is a complete 
representation of $\mathcal{A}$ with the proviso that 1' may not be respected. (Representations not respecting 1' have been considered earlier by (e.g.) Jónsson, Tarski, and Comer.) The rough idea is now that any complete representation of $\mathcal{P}_{n}(\mathcal{A})$ over a base set $U$ is a free amalgam of loose representations of $\mathcal{A}$.

In a little more detail, for each subset $Z \subseteq U$ of cardinality $n-3$, there is a loose representation of $\mathcal{A}$ on the base $U \backslash Z$, which we will call the $Z$-representation, regarded in 3-dimensional cylindric (and in fact polyadic) fashion in the same way as in (ii) above. There is no connection between the loose $Z$-representations for different $Z$.

$\mathcal{P}_{n}(\mathcal{A})$ is an $n$-dimensional algebra and each element of it is interpreted as an $n$-ary relation on $U$ - a set of $n$-tuples. The relations in $\mathcal{P}_{n}(\mathcal{A})$ that 'hold' on a given tuple $\bar{a} \in{ }^{n} U$ embody what $\bar{a}$ can 'see' in the representation. Plainly, $\bar{a}$ is 'aware' of the $Z$-representation for each $(n-3)$-sized set $Z \subseteq \operatorname{rng} \bar{a}$, if any: it can directly inspect the (at most three) elements of $(\operatorname{rng} \bar{a}) \backslash Z$, and using cylindrifications it can 'see' other elements of $U \backslash Z$ in this same $Z$-representation. Now a loose representation of $\mathcal{A}$ respects only the relation algebra operations and may not survive inspection at dimensions higher than three - see the example in $[17,18]$. But $\bar{a}$ can never 'see' more than three elements of the $Z$-representation at once, because this would entail 'moving' some element of $\operatorname{rng} \bar{a} \cap Z$, at which point $Z$ is no longer a subset of the tuple and all information about the $Z$-representation is lost.

A tuple $\bar{a}$ may also 'see' elements of other $Z$-representations, for other $(n-3)$-sized sets $Z \subseteq \operatorname{rng} \bar{a}$. But these representations are independent of one another, because the representation of $\mathcal{P}_{n}(\mathcal{A})$ is a free amalgam of loose representations of $\mathcal{A}$. (This amalgamation is done with a game, and is why loose representations of $\mathcal{A}$ are needed: e.g., we could not amalgamate a finite representation with an infinite one.) Any interaction between the $Z$-representations for different $Z$ is coincidental and not dangerous.

That is the rough idea: now we have to build $\mathcal{P}_{n}(\mathcal{A})$ to make it work. Each atom of $\mathcal{P}_{n}(\mathcal{A})$ will be a whole cluster of atomic $\mathcal{A}$-networks of dimension at most three, each with domain a subset of $n$ whose complement corresponds to an $(n-3)$-sized subset of the range of any tuple satisfying the atom. Complete representability of $\mathcal{A}$ will be preserved and reflected by $\mathcal{P}_{n}(\mathcal{A})$ because (i) much as in the 3 -dimensional case, a complete representation of $\mathcal{A}$ can be 'read off' from any of the loose representations in a complete representation of $\mathcal{P}_{n}(\mathcal{A})$ (see Proposition 4.12), and (ii) a complete representation of $\mathcal{P}_{n}(\mathcal{A})$ can be constructed over an infinite base set $U$ by freely amalgamating loose representations of $\mathcal{A}$, indexed by sets $Z$ of $n-3$ distinct points of $U$ (see Proposition 4.7).

We remark that contrary to possible expectations, and differently from both Monk's and Maddux's constructions $[20,16], \mathcal{A}$ is not in general isomorphic to the relation algebra reduct of $\mathcal{P}_{n}(\mathcal{A})$. (For example, if $n \geq 6$ and $\mathcal{A}$ is 
representable then $\left\{\mathrm{c}_{2} \cdots \mathrm{c}_{n-1} \nu: \nu \in \mathcal{S}_{n}(\mathcal{A})\right\} \subseteq\left\{\mathrm{d}_{01},-\mathrm{d}_{01}\right\}$. So the relation algebra reduct of $\mathcal{P}_{n}(\mathcal{A})$ is just $\left\{0,1, \mathrm{~d}_{01},-\mathrm{d}_{01}\right\}$.) This is unsurprising, considering the form of representations of $\mathcal{P}_{n}(\mathcal{A})$.

In Subsections 3.1-3.2, we formally present our construction. In Section 4, we will show that the construction preserves and reflects complete representability. Fix a simple atomic relation algebra $\mathcal{A}$.

\subsection{The atom structure $\mathcal{S}_{n}(\mathcal{A})$.}

Definition 3.1. We will let $\mathcal{S}=\mathcal{S}_{n}(\mathcal{A})$ denote the following $n$-dimensional polyadic equality atom structure. Its elements (atoms) are the families of the form

$$
\nu=\left(\sim^{\nu}, N_{X}^{\nu}: X \in H\left(\sim^{\nu}\right)\right),
$$

where $\sim^{\nu} \in E q(n)$ and for each $X \in H\left(\sim^{\nu}\right), N_{X}^{\nu}$ is an atomic $\mathcal{A}$-network with domain $X$ and such that for each $i, j \in X$, if $i \sim^{\nu} j$, then $N_{X}^{\nu}(i, j) \leq 1$ ' note that the converse implication need not hold). For $i, j<n$ and $\sigma: n \rightarrow n$, we define

(1) $\mathrm{d}_{i j}=\left\{\nu \in \mathcal{S}: i \sim^{\nu} j\right\}$

(2) for $\nu, \nu^{\prime} \in \mathcal{S}, \nu \equiv_{i} \nu^{\prime}$ iff $\sim^{\nu}={ }_{i} \sim^{\nu^{\prime}}$ and $N_{X}^{\nu}={ }_{i} N_{X^{\prime}}^{\nu^{\prime}}$ whenever $X \in H\left(\sim^{\nu}\right)$ and $X^{\prime} \in H\left(\sim^{\nu^{\prime}}\right)$ match for $\sim^{\nu}$ off $i$,

(3) for $\nu \in \mathcal{S}, \nu^{\sigma}=\left(\left(\sim^{\nu}\right)_{\sigma}, N_{X}^{\nu^{\sigma}}: X \in H\left(\left(\sim^{\nu}\right)_{\sigma}\right)\right)$, where $N_{X}^{\nu^{\sigma}}$ is defined informally by ' $N_{\sigma_{\nu}(X)}^{\nu} \circ \sigma$ ' and formally by

$$
\begin{aligned}
N_{X}^{\nu^{\sigma}}(i, j) & =N_{\sigma_{\nu}(X)}^{\nu}(\sigma(i), \sigma(j)) & & \text { for } i, j \in X, \text { where } \\
\sigma_{\nu}(X) & =\sigma_{\sim^{\nu}}(X) & & \text { (see Definition 2.8). }
\end{aligned}
$$

As rough intuition, suppose that the complex algebra over $\mathcal{S}_{n}(\mathcal{A})$ has a complete (hence atomic) square representation over the base set $U$. For each $Z \subseteq U$ of size $n-3$, there should be a loose representation of $\mathcal{A}$ on $U \backslash Z$. For an $n$-tuple $\bar{a} \in{ }^{n} U$ to stand in the relation $\nu$, we intend firstly that $a_{i}=a_{j}$ iff $i \sim^{\nu} j$ for each $i, j<n$. In consequence, $H\left(\sim^{\nu}\right)$ is the set of all $X \subseteq n$ such that the set $Z=\left\{a_{i}: i \in n \backslash X\right\}$ has exactly $n-3$ elements, and $a_{i} \in U \backslash Z$ for each $i \in X$. For each $i, j \in X \in H\left(\sim^{\nu}\right)$, the atom $N_{X}^{\nu}(i, j)$ of $\mathcal{A}$ is interpreted as a binary relation in the loose representation of $\mathcal{A}$ on $U \backslash Z$. We intend secondly that $\left(a_{i}, a_{j}\right)$ lies in this relation. On this view, it is not so hard to see that if $\bar{a}, \bar{a}^{\prime} \in{ }^{n} U$ are in the relations $\nu, \nu^{\prime}$, respectively, $i, j<n$, and $\sigma: n \rightarrow n$, then $a_{i}=a_{j}$ iff $\nu \in \mathrm{d}_{i j}, \bar{a}={ }_{i} \bar{a}^{\prime} \Rightarrow \nu \equiv_{i} \nu^{\prime}$, and if $\bar{a}^{\prime}=\bar{a} \circ \sigma$, then $\nu^{\prime}=\nu^{\sigma}$. The details are in Lemma 4.3.

We now check that $\mathcal{S}_{n}(\mathcal{A})$ is a well-defined polyadic equality atom structure. We will write $\sim$ for $\sim^{\nu}$ if the context makes the meaning clear. Recall that Id denotes the identity map on $n$.

Lemma 3.2. For each $i<n, \equiv_{i}$ in Definition 3.1 is an equivalence relation.

Proof. We check that $\equiv_{i}$ is reflexive. Suppose $\nu=\nu^{\prime}$ in the definition. Then $\sim^{\nu}=\sim^{\nu^{\prime}}=\sim$, say, so $\sim^{\nu}={ }_{i} \sim^{\nu^{\prime}}$. If $X, X^{\prime} \in H(\sim)$ match for $\sim$ off $i$, 
then $X=\operatorname{Id}_{\sim}(X \cup\{i\})=\operatorname{Id}_{\sim}\left(X^{\prime} \cup\{i\}\right)=X^{\prime}$ by Lemma 2.9(3), so certainly $N_{X}^{\nu}={ }_{i} N_{X^{\prime}}^{\nu^{\prime}}$. That $\equiv_{i}$ is symmetric follows from Lemma 2.9(2).

We check transitivity. Suppose $\nu \equiv_{i} \nu^{\prime} \equiv_{i} \nu^{\prime \prime}$. Write $\sim^{\nu}=\sim, \sim^{\nu^{\prime}}=\sim^{\prime}$, etc. Then $\sim={ }_{i} \sim^{\prime}={ }_{i} \sim^{\prime \prime}$, so certainly $\sim={ }_{i} \sim^{\prime \prime}$. Let $X \in H(\sim)$ and $X^{\prime \prime} \in H\left(\sim^{\prime \prime}\right)$ match for $\sim$ off $i$, so $X \cup\{i\}=X^{\prime \prime} \cup\{i\}=I$, say, and $|(n \backslash I) / \sim|=n-3$. Since $\sim={ }_{i} \sim^{\prime}$ and $i \in I$, we have $\left|(n \backslash I) / \sim^{\prime}\right|=n-3$, and so $X^{\prime}=n \backslash \bigcup\left((n \backslash I) / \sim^{\prime}\right) \in$ $H\left(\sim^{\prime}\right)$. Clearly, $I=X^{\prime} \cup\{i\}$ as well, so $X, X^{\prime}$ match for $\sim$ off $i$ and $X^{\prime}, X^{\prime \prime}$ match for $\sim^{\prime}$ off $i$. By hypothesis, $N_{X}^{\nu}={ }_{i} N_{X^{\prime}}^{\nu^{\prime}}={ }_{i} N_{X^{\prime \prime}}^{\nu^{\prime \prime}}$, yielding $N_{X}^{\nu}={ }_{i} N_{X^{\prime \prime}}^{\nu^{\prime \prime}}$, as required.

Lemma 3.3. For each $\sigma: n \rightarrow n$ and $\nu \in \mathcal{S}_{n}(\mathcal{A})$, the atom $\nu^{\sigma}$ is well defined and in $\mathcal{S}_{n}(\mathcal{A})$.

Proof. Write $\sim$ for $\sim^{\nu}$. Then $\sim^{\nu^{\sigma}}=\sim_{\sigma} \in E q(n)$. Let $X \in H\left(\sim_{\sigma}\right)$. By Lemma 2.9(5c), $\sigma_{\nu}(X) \in H(\sim)$, and so $N_{\sigma_{\nu}(X)}^{\nu}$ is defined. By Lemma 2.9(5a), $\sigma[X] \subseteq \sigma_{\nu}(X)$, so for any $i, j \in X, N_{\sigma_{\nu}(X)}^{\nu}(\sigma(i), \sigma(j))$ is well defined. So $N_{X}^{\nu^{\sigma}}$ is well defined, and clearly it is an atomic $\mathcal{A}$-network. Finally, if $i \sim^{\nu^{\sigma}} j$, then by definition, $i \sim_{\sigma} j$, so $\sigma(i) \sim \sigma(j)$, and $N_{X}^{\nu^{\sigma}}(i, j)=N_{\sigma_{\nu}(X)}^{\nu}(\sigma(i), \sigma(j)) \leq 1$, as required.

Lemma 3.4. $\mathcal{S}_{n}(\mathcal{A})$ is a polyadic equality atom structure.

Proof. By Lemma 3.2, each $\equiv_{i}$ is an equivalence relation. Writing $\sigma \tau$ for $\sigma \circ \tau$, we check that $\nu^{\sigma \tau}=\left(\nu^{\sigma}\right)^{\tau}$ for each $\nu, \sigma, \tau$. Again, write $\sim$ for $\sim^{\nu}$. First observe that $i \sim_{\sigma \tau} j$ iff $\sigma(\tau(i)) \sim \sigma(\tau(j))$, iff $\tau(i) \sim_{\sigma} \tau(j)$, iff $i\left(\sim_{\sigma}\right)_{\tau} j$. So $\left(\sim_{\sigma}\right)_{\tau}=\sim_{\sigma \tau}$ - the equivalence relations in $\left(\nu^{\sigma}\right)^{\tau}$ and $\nu^{\sigma \tau}$ are the same. It follows that $H\left(\left(\sim_{\sigma}\right)_{\tau}\right)=H\left(\sim_{\sigma \tau}\right)$, so the sets of indices of networks in $\left(\nu^{\sigma}\right)^{\tau}$ and $\nu^{\sigma \tau}$ are the same as well. Now we take arbitrary $X$ in this set and show that $N_{X}^{\left(\nu^{\sigma}\right)^{\tau}}=N_{X}^{\nu^{\sigma \tau}}$. Informally, it follows from the definitions that

$$
N_{X}^{\left(\nu^{\sigma}\right)^{\tau}}=N_{\tau_{\nu}(X)}^{\nu^{\sigma}} \circ \tau=\left(N_{\sigma_{\nu}\left(\tau_{\nu} \sigma(X)\right)}^{\nu} \circ \sigma\right) \circ \tau=N_{(\sigma \circ \tau) \nu(X)}^{\nu} \circ(\sigma \tau)=N_{X}^{\nu^{\sigma \tau}},
$$

as required (that $\sigma_{\nu}\left(\tau_{\nu^{\sigma}}(X)\right)=(\sigma \tau)_{\nu}(X)$ follows from Lemma 2.9(6)). The networks here are well defined by Lemma 3.3 .

\subsection{The algebra $\mathcal{P}_{n}(\mathcal{A})$.}

Definition 3.5. We write $\mathcal{P}_{n}(\mathcal{A})$ for the complex algebra $\mathcal{S}_{n}(\mathcal{A})^{+}$.

$\mathcal{P}_{n}(\mathcal{A})$ is an atomic $n$-dimensional polyadic equality-type algebra. It is finite iff $\mathcal{A}$ is finite, and in that case, it is effectively constructible from $\mathcal{A}$.

We briefly examine substitutions in $\mathcal{P}_{n}(\mathcal{A})$. For $i, j<n$, write $[i / j]: n \rightarrow n$ for the map given by $[i / j](i)=j$ and $[i / j](k)=k$ for $k \in n \backslash\{i\}$.

Lemma 3.6. Let $i, j<n$ be distinct and let $\nu, \nu^{\prime} \in \mathcal{S}_{n}(\mathcal{A})$. Then $\nu^{\prime}=\nu^{[i / j]}$ iff $\nu \equiv_{i} \nu^{\prime}$ and $\nu^{\prime} \in \mathrm{d}_{i j}$. Hence, $\mathcal{P}_{n}(\mathcal{A}) \models \forall x\left(\mathrm{~s}_{[i / j]} x=\mathrm{c}_{i}\left(\mathrm{~d}_{i j} \cdot x\right)\right)$. 
Proof. Write $\sigma=[i / j]$ and $\sim=\sim^{\nu}$. Recall $\sim^{\sigma}=\sim_{\sigma}$. Plainly, $\sigma(i)=$ $\sigma(j)=j$, so $i \sim_{\sigma} j$, and hence $\nu^{\sigma} \in \mathrm{d}_{i j}$. We check that $\nu \equiv_{i} \nu^{\sigma}$. Certainly, $\sim_{\sigma}={ }_{i} \sim$. Let $X \in H(\sim), Y \in H\left(\sim_{\sigma}\right)$, and suppose $X$ and $Y$ match for $\sim$ off $i$, so $X \cup\{i\}=Y \cup\{i\}=I$, say, and $|(n \backslash I) / \sim|=n-3$. We require $N_{X}^{\nu}={ }_{i} N_{Y}^{\nu^{\sigma}}$. Certainly, $X \backslash\{i\}=Y \backslash\{i\}$. Let $k, l$ lie in this set; we check that $N_{X}^{\nu}(k, l)=N_{Y}^{\nu^{\sigma}}(k, l)$. Now $N_{Y}^{\nu^{\sigma}}(k, l)=N_{\sigma_{\nu}(Y)}^{\nu}(\sigma(k), \sigma(l))=N_{\sigma_{\sim}(Y)}^{\nu}(k, l)$. But by Lemma $2.9(3,6,4), \sigma_{\sim}(Y)=\sigma_{\sim}\left(\operatorname{Id}_{\sim_{\sigma}}(I)\right)=(\sigma \circ \mathrm{Id}) \sim(I)=\sigma_{\sim}(I)=$ $I d_{\sim}(I)=X$.

For the converse, assume that $\nu \equiv_{i} \nu^{\prime} \in \mathrm{d}_{i j}$. We require $\nu^{\prime}=\nu^{\sigma}$. As usual, we write $\sim^{\prime}$ for $\sim^{\nu^{\prime}}$. First, from the assumptions we obtain $\sim^{\prime}={ }_{i} \sim$ and $i \sim^{\prime} j$. It follows that $\sim^{\prime}=\sim_{\sigma}$. Now let $X \in H\left(\sim^{\prime}\right)=H\left(\sim_{\sigma}\right)$. We require $N_{X}^{\nu^{\prime}}=N_{X}^{\nu^{\sigma}}$

Claim 1. For all $m<n$ we have $m \in X$ iff $\sigma(m) \in X$, and in that case, $N_{X}^{\nu^{\prime}}(\sigma(m), m) \leq 1$ ' and $N_{X}^{\nu^{\prime}}(m, \sigma(m)) \leq 1$ '.

Proof of claim. As $\sigma=\sigma \circ \sigma$, we have $m \sim_{\sigma} \sigma(m)$. The first part follows since $X$ is a union of $\sim_{\sigma}$-classes, and the second by definition of $\mathcal{S}_{n}(\mathcal{A})$ since $m \sim^{\prime} \sigma(m)$.

To prove that $N_{X}^{\nu^{\prime}}=N_{X}^{\nu^{\sigma}}$, we take arbitrary $k, l \in X$ and prove that $N_{X}^{\nu^{\prime}}(k, l)=N_{X}^{\nu^{\sigma}}(k, l)$. By definition,

$$
N_{X}^{\nu^{\sigma}}(k, l)=N_{\sigma_{\nu}(X)}^{\nu}(\sigma(k), \sigma(l)) .
$$

Claim 2. $\sigma_{\nu}(X)$ and $X$ match for $\sim$ off $i$.

Proof of claim. By Lemma 2.9(5),

$$
X \backslash\{i\} \subseteq \sigma[X] \subseteq \sigma_{\sim}(X) \text { and } n \backslash(X \cup\{i\}) \subseteq \sigma[n \backslash X] \subseteq n \backslash \sigma_{\sim}(X) .
$$

It follows that $X \backslash\{i\}=\sigma_{\nu}(X) \backslash\{i\}$, and so $X \cup\{i\}=\sigma_{\nu}(X) \cup\{i\}=I$, say. By Claim 1, $i \in X$ iff $j=\sigma(i) \in X$. As $j \neq i$, this is iff $j \in I$, and it follows that $\sigma[n \backslash X]=n \backslash I$. So by Lemma 2.9(5b), $(n \backslash I) / \sim=(\sigma[n \backslash X]) / \sim=$ $\left(n \backslash \sigma_{\sim}(X)\right) / \sim$, and the latter set has size $n-3$ by Lemma $2.9(5 \mathrm{c})$. This proves the claim.

Since $\nu \equiv_{i} \nu^{\prime}$, Claim 2 yields $N_{\sigma_{\nu}(X)}^{\nu}={ }_{i} N_{X}^{\nu^{\prime}}$. As $i \notin \operatorname{rng} \sigma$, we get

$$
N_{\sigma_{\nu}(X)}^{\nu}(\sigma(k), \sigma(l))=N_{X}^{\nu^{\prime}}(\sigma(k), \sigma(l)) .
$$

By networkhood of $N_{X}^{\nu^{\prime}}$ and Claim 1,

$$
\begin{aligned}
N_{X}^{\nu^{\prime}}(\sigma(k), \sigma(l)) & \leq N_{X}^{\nu^{\prime}}(\sigma(k), k) ; N_{X}^{\nu^{\prime}}(k, l) ; N_{X}^{\nu^{\prime}}(l, \sigma(l)) \\
& \leq 1^{\prime} ; N_{X}^{\nu^{\prime}}(k, l) ; 1^{\prime}=N_{X}^{\nu^{\prime}}(k, l),
\end{aligned}
$$

and since both are atoms of $\mathcal{A}$,

$$
N_{X}^{\nu^{\prime}}(\sigma(k), \sigma(l))=N_{X}^{\nu^{\prime}}(k, l) .
$$

From (3.3), (3.4), and (3.5), we obtain $N_{X}^{\nu^{\sigma}}(k, l)=N_{X}^{\nu^{\prime}}(k, l)$ as required.

For the second part of the lemma, for any $r \in \mathcal{P}_{n}(\mathcal{A})$ we have 


$$
\begin{aligned}
\mathrm{s}_{[i / j]} r=\left\{\nu \in \mathcal{S}_{n}(\mathcal{A}): \nu^{[i / j]} \in r\right\} & \\
& =\left\{\nu \in \mathcal{S}_{n}(\mathcal{A}): \exists \nu^{\prime}\left(\nu \equiv_{i} \nu^{\prime} \wedge \nu^{\prime} \in \mathrm{d}_{i j} \cap r\right)\right\}=\mathrm{c}_{i}\left(\mathrm{~d}_{i j} \cdot r\right),
\end{aligned}
$$

as required.

\section{Representations}

In this section, we prove that for any subsignature $L$ of the signature of $\mathcal{P}_{n}(\mathcal{A})$ containing the booleans and cylindrifications, $\mathcal{A}$ is completely representable iff the $L$-reduct of $\mathcal{P}_{n}(\mathcal{A})$ is completely representable. The proof proceeds in a circle. Assuming that $\mathcal{A}$ is completely representable, we show that $\mathcal{P}_{n}(\mathcal{A})$ has a complete representation by constructing one using a game. It is trivial that if $\mathcal{P}_{n}(\mathcal{A})$ is completely representable, then so is its $L$-reduct, and if its $L$-reduct is completely representable, then so is the reduct of $\mathcal{P}_{n}(\mathcal{A})$ to the smallest signature: that of diagonal-free cylindric algebras. Extending a result of Johnson, we show that if this reduct of $\mathcal{P}_{n}(\mathcal{A})$ is completely representable, then so is its cylindric reduct, and if this is completely representable, then we can read off a complete representation of $\mathcal{A}$. This will all be done in Subsection 4.2. The next subsection contains some preliminary work needed for the game argument.

4.1. Networks from representations. Our games will be played on $\mathcal{S}_{n}(\mathcal{A})$ networks.

Definition 4.1. Let $\mathcal{S}$ be any $n$-dimensional polyadic equality atom structure. An $\mathcal{S}$-network is a structure $M=\left(M_{1}, M_{2}\right)$, where $M_{1}$ is a (possibly empty) set and $M_{2}:{ }^{n} M_{1} \rightarrow \mathcal{S}$ satisfies that for all $\bar{a}, \bar{b} \in{ }^{n} M_{1}, i, j<n$, and $\sigma: n \rightarrow n$,

(1) $M_{2}(\bar{a}) \in \mathrm{d}_{i j} \Longleftrightarrow a_{i}=a_{j}$,

(2) $\bar{a}={ }_{i} \bar{b} \Longrightarrow M_{2}(\bar{a}) \equiv_{i} M_{2}(\bar{b})$,

(3) $M_{2}(\bar{a} \circ \sigma)=M_{2}(\bar{a})^{\sigma}$.

For $\mathcal{S}$-networks $M=\left(M_{1}, M_{2}\right)$ and $M^{\prime}=\left(M_{1}^{\prime}, M_{2}^{\prime}\right)$, we write $M \subseteq M^{\prime}$ if $M_{i} \subseteq M_{i}^{\prime}$ for each $i=1,2$. As with relation algebra networks, we may write $M$ for any of $M, M_{1}, M_{2}$, and we will call $M_{1}$ the domain of $M$, or its set of nodes.

We now show how to build $\mathcal{S}_{n}(\mathcal{A})$-networks from a complete representation of $\mathcal{A}$. Until the end of Subsection 4.1, suppose that $\mathcal{A}$ is completely representable. Then, being simple, it has a 'square' complete representation $h: \mathcal{A} \rightarrow \mathfrak{R e}\left(R_{1}\right)$ for some set $R_{1}$, where (recall from Definition 2.2) $\mathfrak{R e}\left(R_{1}\right)$ denotes the algebra of all binary relations on $R_{1}$. We can view $h$ as a strict atomic $\mathcal{A}$-network $R=\left(R_{1}, R_{2}\right)$, where

$$
R_{2}(x, y)=\prod\{a \in \mathcal{A}:(x, y) \in h(a)\} \in \operatorname{At} \mathcal{A}, \quad \text { for each } x, y \in R_{1} .
$$

(By Remark 2.5, this is indeed an atom of $\mathcal{A}$, and one can check that $R$ is a strict atomic $\mathcal{A}$-network.) 
For a set $X$, and $m<\omega$, we let $[X]^{m}=\{Y \subseteq X:|Y|=m\}$ as usual.

Definition 4.2. Let $M_{1}$ be a non-empty set and $\mathcal{F}=\left\{f_{Z}: Z \in\left[M_{1}\right]^{n-3}\right\}$ a set of maps, where $f_{Z}: M_{1} \backslash Z \rightarrow R$ for each $Z$. The maps have no special properties and there need be no connection between them. For each $\bar{a} \in{ }^{n} M_{1}$, we define an atom $M_{2}(\bar{a})=\nu=\left(\sim, N_{X}^{\nu}: X \in H(\sim)\right) \in \mathcal{S}_{n}(\mathcal{A})$ as follows.

- $i \sim j \Longleftrightarrow a_{i}=a_{j}$ for $i, j<n$,

- for $X \in H(\sim)$, we observe that $Z=\left\{a_{i}: i \in n \backslash X\right\} \in\left[M_{1}\right]^{n-3}$, and define $N_{X}^{\nu}(i, j)=R\left(f_{Z}\left(a_{i}\right), f_{Z}\left(a_{j}\right)\right)$ for $i, j \in X$.

We define $\operatorname{Net}\left(M_{1}, \mathcal{F}\right)=\left(M_{1}, M_{2}\right)$.

In the second bullet point above, $a_{i}, a_{j} \in M_{1} \backslash Z$ by definition of $\sim$ and $H(\sim)$, and so $f_{Z}\left(a_{i}\right), f_{Z}\left(a_{j}\right)$ are defined. Clearly, $N_{X}^{\nu}$ is an atomic $\mathcal{A}$-network with domain $X$. Moreover, if $i, j \in X$, then $i \sim j \Rightarrow a_{i}=a_{j} \Rightarrow N_{X}^{\nu}(i, j) \leq 1^{\prime}$, as required. So indeed $\nu \in \mathcal{S}_{n}(\mathcal{A})$.

Lemma 4.3. $\operatorname{Net}\left(M_{1}, \mathcal{F}\right)$ is an $\mathcal{S}_{n}(\mathcal{A})$-network.

Proof. Write $M$ for $\operatorname{Net}\left(M_{1}, \mathcal{F}\right)$. Let $\bar{a} \in{ }^{n} M$ and $M(\bar{a})=\nu$. Then for $i, j<n$, we have $\nu \in \mathrm{d}_{i j}$ iff $i \sim^{\nu} j$ iff $a_{i}=a_{j}$, as required.

Next let $i<n$ and $\bar{a}, \bar{b} \in{ }^{n} M$ with $\bar{a}={ }_{i} \bar{b}$. Suppose $M(\bar{a})=\nu$ and $M(\bar{b})=\nu^{\prime}$. We require $\nu \equiv_{i} \nu^{\prime}$. As usual, we write $\sim$ for $\sim^{\nu}$ and $\sim^{\prime}$ for $\sim^{\nu^{\prime}}$. Certainly, $\sim={ }_{i} \sim^{\prime}$. Take $X \in H(\sim)$ and $X^{\prime} \in H\left(\sim^{\prime}\right)$ that match for $\sim$ off $i$. So $X \cup\{i\}=X^{\prime} \cup\{i\}=I$, say, and $|(n \backslash I) / \sim|=n-3$. We desire $N_{X}^{\nu}={ }_{i} N_{X^{\prime}}^{\nu^{\prime}}$. Clearly, $X \backslash\{i\}=X^{\prime} \backslash\{i\}$. Moreover, $(n \backslash X) / \sim=(n \backslash I) / \sim$ and $(n \backslash I) / \sim^{\prime}=\left(n \backslash X^{\prime}\right) / \sim^{\prime}$ since $X, X^{\prime} \subseteq I \ni i$ and these sets of equivalence classes all have size $n-3$. Let $Z=\left\{a_{j}: j \in n \backslash X\right\}$ and $Z^{\prime}=\left\{b_{j}: j \in n \backslash X^{\prime}\right\}$. These sets have size $n-3$. Moreover,

$$
\begin{aligned}
Z & =\left\{a_{j}: j \in n \backslash I\right\} & & \text { because }(n \backslash X) / \sim=(n \backslash I) / \sim, \\
& =\left\{b_{j}: j \in n \backslash I\right\} & & \text { because } \bar{a}={ }_{i} \bar{b} \text { and } i \in I, \\
& =Z^{\prime} & & \text { because }(n \backslash I) / \sim^{\prime}=(n \backslash X) / \sim^{\prime} .
\end{aligned}
$$

Now take any $j, k \in X \backslash\{i\}$. By definition of $\operatorname{Net}\left(M_{1}, \mathcal{F}\right)$, we have

$$
N_{X}^{\nu}(j, k)=R\left(f_{Z}\left(a_{j}\right), f_{Z}\left(a_{k}\right)\right)=R\left(f_{Z^{\prime}}\left(b_{j}\right), f_{Z^{\prime}}\left(b_{k}\right)\right)=N_{X^{\prime}}^{\nu^{\prime}}(j, k),
$$

as required to prove $\nu \equiv_{i} \nu^{\prime}$.

Finally, take $\bar{a} \in{ }^{n} M$ and $\sigma: n \rightarrow n$. Let $M(\bar{a})=\nu$ and $M(\bar{a} \circ \sigma)=\nu^{\prime}$, say. We require $\nu^{\prime}=\nu^{\sigma}$. We adopt the usual abbreviations. Obviously, $i \sim^{\prime} j$ iff $(\bar{a} \circ \sigma)_{i}=(\bar{a} \circ \sigma)_{j}$ iff $a_{\sigma(i)}=a_{\sigma(j)}$ iff $\sigma(i) \sim \sigma(j)$ iff $i \sim_{\sigma} j$ for $i, j<n$; so $\sim^{\prime}=\sim_{\sigma}$. Hence, $H\left(\sim^{\prime}\right)=H\left(\sim_{\sigma}\right)$. Take $X$ in this set. We require $N_{X}^{\nu^{\prime}}=N_{X}^{\nu^{\sigma}}$. Let $Z=\left\{a_{i}: i \in n \backslash \sigma_{\nu}(X)\right\}$ and $Z^{\prime}=\left\{(\bar{a} \circ \sigma)_{i}: i \in n \backslash X\right\} \in[M]^{n-3}$. It can be seen by Lemma $2.9(5 \mathrm{~b})$ and the definition of $\sim$ that $Z=Z^{\prime}$. Let $i, j \in X$. By Lemma 2.9(5a), $\sigma(i), \sigma(j) \in \sigma_{\nu}(X)$. By definition of $\operatorname{Net}\left(M_{1}, \mathcal{F}\right)$, we have $N_{X}^{\nu^{\prime}}(i, j)=R\left(f_{Z^{\prime}}\left((\bar{a} \circ \sigma)_{i}\right), f_{Z^{\prime}}\left((\bar{a} \circ \sigma)_{j}\right)\right)=R\left(f_{Z}\left(a_{\sigma(i)}\right), f_{Z}\left(a_{\sigma(j)}\right)\right)=$ $N_{\sigma_{\nu}(X)}^{\nu}(\sigma(i), \sigma(j))=N_{X}^{\nu^{\sigma}}(i, j)$, as required. 
So we can make an $\mathcal{S}_{n}(\mathcal{A})$-network from a set of maps. There is a kind of converse to this for very small $\mathcal{S}_{n}(\mathcal{A})$-networks: see Lemma 4.6.

Definition 4.4. Let $N, N^{\prime}$ be atomic $\mathcal{A}$-networks. A partial map $f: N \rightarrow N^{\prime}$ is said to be a weak embedding if $N(i, j)=N^{\prime}(f(i), f(j))$ for all $i, j \in \operatorname{dom} f$. (We do not require that $f$ is $1-1$.)

The following is easily proved using basic properties of relation algebras:

Lemma 4.5. Let $N$ be any atomic $\mathcal{A}$-network with at most three nodes. Then any partial weak embedding $h_{0}: N \rightarrow R$ extends to a total weak embedding $h: N \rightarrow R$.

Lemma 4.6. Let $\nu=\left(\sim, N_{X}^{\nu}: X \in H(\sim)\right) \in \mathcal{S}_{n}(\mathcal{A})$ be given, and let $\bar{a}=$ $\left(a_{0}, \ldots, a_{n-1}\right)$ be a tuple such that $a_{i}=a_{j}$ iff $i \sim j$ for $i, j<n$. Write $M_{1}=\operatorname{rng} \bar{a}$. For each $Z \in\left[M_{1}\right]^{n-3}$, let $h_{Z}^{0}: M_{1} \backslash Z \rightarrow R$ be a partial map, and suppose that for each $X \in H(\sim)$, if $Z=\left\{a_{i}: i \in n \backslash X\right\}$, then the map

$$
g_{Z}^{0}=h_{Z}^{0} \circ \bar{a} \uparrow X: N_{X}^{\nu} \rightarrow R
$$

is a partial weak embedding. Then there is a set $\mathcal{H}=\left\{h_{Z}: Z \in\left[M_{1}\right]^{n-3}\right\}$, where $h_{Z}: M_{1} \backslash Z \rightarrow R$ is a total map extending $h_{Z}^{0}$ (for each $Z$ ), such that, writing $M=\operatorname{Net}\left(M_{1}, \mathcal{H}\right)$, we have $M(\bar{a})=\nu$.

Proof. For each $Z \in\left[M_{1}\right]^{n-3}$, select the unique $X \in H(\sim)$ such that $Z=$ $\left\{a_{i}: i \in n \backslash X\right\}$. Using Lemma 4.5, extend $g_{Z}^{0}$ to a total weak embedding $g_{Z}: N_{X}^{\nu} \rightarrow R$. Now define $h_{Z}: M_{1} \backslash Z \rightarrow R$ by $h_{Z}\left(a_{i}\right)=g_{Z}(i)$ for $i \in X$. This is well defined. For if $i, j \in X$ and $a_{i}=a_{j}$, then $i \sim j$, so as $\nu \in \mathcal{S}_{n}(\mathcal{A})$, we have $N_{X}^{\nu}(i, j) \leq 1$ '. Hence, $R\left(g_{Z}(i), g_{Z}(j)\right) \leq 1^{\prime}$, and so $g_{Z}(i)=g_{Z}(j)$ as $R$ is strict. Plainly, $h_{Z}$ is total and extends $h_{Z}^{0}$.

We now define $\mathcal{H}=\left\{h_{Z}: Z \in\left[M_{1}\right]^{n-3}\right\}, M=\operatorname{Net}\left(M_{1}, \mathcal{H}\right)$, and $\nu^{\prime}=M(\bar{a})$. We check that $\nu^{\prime}=\nu$. Certainly, $\sim^{\prime}=\sim$. Let $X \in H(\sim)$ and $i, j \in X$. Put $Z=\left\{a_{k}: k \in n \backslash X\right\} \in\left[M_{1}\right]^{n-3}$. Then

$$
\begin{aligned}
N_{X}^{\nu^{\prime}}(i, j) & =R\left(h_{Z}\left(a_{i}\right), h_{Z}\left(a_{j}\right)\right) & & \text { by definition of } \operatorname{Net}\left(M_{1}, \mathcal{H}\right), \\
& =R\left(g_{Z}(i), g_{Z}(j)\right) & & \text { by definition of } h_{Z}, \\
& =N_{X}^{\nu}(i, j) & & \text { as } g_{Z}: N_{X}^{\nu} \rightarrow R \text { is a weak embedding. }
\end{aligned}
$$

Hence, $N_{X}^{\nu^{\prime}}=N_{X}^{\nu}$ for each $X \in H(\sim)$, showing that $\nu^{\prime}=\nu$.

4.2. Building representations. This subsection contains the main technical results of the paper. We will prove 'equi-complete representability' of $\mathcal{A}$ and all 'pseudo-diagonal-free reducts' of $\mathcal{P}_{n}(\mathcal{A})$.

Proposition 4.7. Suppose that $\mathcal{A}$ has a complete representation. Then $\mathcal{P}_{n}(\mathcal{A})$ has a complete representation as well.

Proof. Let $R$ be a complete representation of $\mathcal{A}$, viewed as in (4.1) above as an atomic $\mathcal{A}$-network. We write $\mathcal{S}=\mathcal{S}_{n}(\mathcal{A})$. Let $\kappa=|\mathcal{S}|+\omega=\mid$ At $\mathcal{A} \mid+\omega$. Players $\forall$ and $\exists$ will play a $\kappa$-round game to build a complete representation 
of $\mathcal{P}_{n}(\mathcal{A})=\mathcal{S}^{+}$in the form of an $\mathcal{S}$-network. There will be an initial round, followed by rounds numbered $0,1, \ldots, t, \ldots(t<\kappa)$. At the start of each numbered round $t<\kappa$, there will be given a pair $\left(D_{t}, \mathcal{F}_{t}\right)$, where $D_{t}$ is a nonempty set and $\mathcal{F}_{t}$ is a set of maps $f_{Z}^{t}: D_{t} \backslash Z \rightarrow R$ (for each $Z \in\left[D_{t}\right]^{n-3}$ ), satisfying

$$
D_{u} \subseteq D_{t} \text { and } f_{Z}^{u} \subseteq f_{Z}^{t} \text { for each } u<t \text { and } Z \in\left[D_{u}\right]^{n-3} .
$$

Consequently, we will have $\operatorname{Net}\left(D_{u}, \mathcal{F}_{u}\right) \subseteq \operatorname{Net}\left(D_{t}, \mathcal{F}_{t}\right)$. Play in round $t$ will create a new set $D_{t+1}$ and a new set of maps $\mathcal{F}_{t+1}=\left\{f_{Z}^{t+1}: Z \in\left[D_{t+1}\right]^{n-3}\right\}$, satisfying (4.3) for $t+1$, and the pair $\left(D_{t+1}, \mathcal{F}_{t+1}\right)$ will be passed to the start of the next round.

In the initial, un-numbered round, $\forall$ chooses an atom $\nu \in \mathcal{S}$. The game rules require that $\exists$ respond with a pair $\left(D_{0}, \mathcal{F}_{0}\right)$ such that if $M=\operatorname{Net}\left(D_{0}, \mathcal{F}_{0}\right)$, then $M(\bar{a})=\nu$ for some $\bar{a} \in{ }^{n} D_{0}$. To do this, she lets $\bar{a}=\left(\{0\} / \sim^{\nu}, \ldots,\{n-1\} / \sim^{\nu}\right)$ (so $a_{i}=a_{j}$ iff $i \sim^{\nu} j$ ), and defines $D_{0}=n / \sim^{\nu}$ and $\mathcal{F}_{0}=\mathcal{H}$ as in Lemma 4.6 (with $h_{Z}^{0}=\emptyset$ for all $Z$ ). By the lemma, $\operatorname{Net}\left(D_{0}, \mathcal{F}_{0}\right)(\bar{a})=\nu$, as required. The pair at the start of round 0 of the main game is defined to be $\left(D_{0}, \mathcal{F}_{0}\right)$. Condition (4.3) holds vacuously.

Let $\left(D_{t}, \mathcal{F}_{t}\right)$ be the pair existing at the start of some numbered round $t$ of the main game $(t<\kappa)$, where $\mathcal{F}_{t}=\left\{f_{Z}^{t}: Z \in\left[D_{t}\right]^{n-3}\right\}$, and (4.3) holds. Write $M=\operatorname{Net}\left(D_{t}, \mathcal{F}_{t}\right)$. Player $\forall$ moves in this round by choosing three items. First, he picks a tuple $\bar{a} \in{ }^{n} D_{t}$. Let $M(\bar{a})=\nu=\left(\sim, N_{X}^{\nu}: X \in H(\sim)\right) \in \mathcal{S}$. Then he chooses an index $i<n$, and an element $\nu^{\prime}=\left(\sim^{\prime}, N_{X}^{\nu^{\prime}}: X \in H\left(\sim^{\prime}\right)\right) \in \mathcal{S}$ satisfying $\nu \equiv_{i} \nu^{\prime}$. He is always able to do this. The rules demand that $\exists$ respond with a pair $\left(D_{t+1}, \mathcal{F}_{t+1}\right)$ satisfying (4.3) for $t+1$ and such that $\operatorname{Net}\left(D_{t+1}, \mathcal{F}_{t+1}\right)(\bar{b})=\nu^{\prime}$ for some $\bar{b} \in{ }^{n} D_{t+1}$ with $\bar{b}={ }_{i} \bar{a}$.

She proceeds as follows. Suppose first that there is $j \in n \backslash\{i\}$ with $\nu^{\prime} \in \mathrm{d}_{i j}$. In that case, by Lemma 3.6, we have $\nu^{\prime}=\nu^{[i / j]}=M(\bar{a} \circ[i / j])$. Then $\exists$ may let $\left(D_{t+1}, \mathcal{F}_{t+1}\right)=\left(D_{t}, \mathcal{F}_{t}\right)$ because she can take $\bar{b}=\bar{a} \circ[i / j]={ }_{i} \bar{a}$.

So suppose not. Now $\exists$ introduces a new node $p \notin D_{t}$, and defines $D_{t+1}=$ $D_{t} \cup\{p\}$. She lets $\bar{b} \in{ }^{n} D_{t+1}$ be given by

$$
b_{j}= \begin{cases}p, & \text { if } j=i, \\ a_{j}, & \text { otherwise, }\end{cases}
$$

for $j<n$. Then $\bar{a}={ }_{i} \bar{b}$. Also, $b_{j}=b_{k}$ iff $j \sim^{\prime} k$, for each $j, k<n$. For if $j, k \neq i$, we have $b_{j}=b_{k}$ iff $a_{j}=a_{k}$ iff $j \sim k$ iff $j \sim^{\prime} k$ (because $\bar{a}={ }_{i} \bar{b}$ and $\nu \equiv_{i} \nu^{\prime}$ ), while $b_{i}=b_{j}$ and $i \sim^{\prime} j$ are both false (because $p \notin D_{t}$ and $\nu^{\prime} \notin \mathrm{d}_{i j}$ ).

Write $B=\operatorname{rng} \bar{b}$. For each $Z \in[B]^{n-3}$, define a partial map $h_{Z}^{0}: B \backslash Z \rightarrow R$ by

$$
h_{Z}^{0}= \begin{cases}f_{Z}^{t} \uparrow\left(D_{t} \cap B \backslash Z\right), & \text { if } Z \subseteq D_{t}, \\ \emptyset, & \text { otherwise (i.e., if } p \in Z) .\end{cases}
$$

Claim. Let $Z \in[B]^{n-3}$ and $X^{\prime}=\left\{j<n: b_{j} \notin Z\right\} \in H\left(\sim^{\prime}\right)$. Then the map $h_{Z}^{0} \circ \bar{b} \uparrow X^{\prime}: N_{X^{\prime}}^{\nu^{\prime}} \rightarrow R$ is a partial weak embedding. 
Proof of claim. The claim holds vacuously if $h_{Z}^{0}=\emptyset$, so assume that $Z \subseteq D_{t}$. Then $Z \subseteq \operatorname{rng} \bar{a}$, and as $|Z|=n-3$, we have $X=\left\{j<n: a_{j} \notin Z\right\} \in H(\sim)$. Since $\bar{a}={ }_{i} \bar{b}$, we have $X \backslash\{i\}=X^{\prime} \backslash\{i\}$, so $X \cup\{i\}=X^{\prime} \cup\{i\}=I$, say. As $b_{i}=p \notin Z$, we have $i \in X^{\prime}$, so $X^{\prime}=I$ and $|(n \backslash I) / \sim|=\left|\left(n \backslash X^{\prime}\right) / \sim\right|=|Z|=$ $n-3$. Thus, $X, X^{\prime}$ match for $\sim$ off $i$. Now $\nu \equiv_{i} \nu^{\prime}$ yields $N_{X}^{\nu}={ }_{i} N_{X^{\prime}}^{\nu^{\prime}}$.

Let $j, k \in X^{\prime}$ with $b_{j}, b_{k} \in \operatorname{dom} h_{Z}^{0}=D_{t} \cap B \backslash Z$. We know $b_{i} \notin D_{t}$, so $j, k \neq i$. Consequently,

$$
\begin{aligned}
N_{X^{\prime}}^{\nu^{\prime}}(j, k) & =N_{X}^{\nu}(j, k) & & \text { since } N_{X}^{\nu}={ }_{i} N_{X^{\prime}}^{\nu^{\prime}} \text { and } j, k \neq i, \\
& =R\left(f_{Z}^{t}\left(a_{j}\right), f_{Z}^{t}\left(a_{k}\right)\right) & & \text { since } M=\operatorname{Net}\left(D_{t}, \mathcal{F}_{t}\right) \text { and } M(\bar{a})=\nu, \\
& =R\left(f_{Z}^{t}\left(b_{j}\right), f_{Z}^{t}\left(b_{k}\right)\right) & & \text { as } \bar{a}={ }_{i} \bar{b} \text { and } j, k \neq i, \\
& =R\left(h_{Z}^{0}\left(b_{j}\right), h_{Z}^{0}\left(b_{k}\right)\right) & & \text { by definition of } h_{Z}^{0},
\end{aligned}
$$

proving the claim.

By the claim and Lemma 4.6, there is a set $\mathcal{H}=\left\{h_{Z}: Z \in[B]^{n-3}\right\}$ of total maps $h_{Z}: B \backslash Z \rightarrow R$ with $h_{Z} \supseteq h_{Z}^{0}$ and $\operatorname{Net}(B, \mathcal{H})(\bar{b})=\nu^{\prime}$. Now, for each $Z \in\left[D_{t+1}\right]^{n-3}, \exists$ defines a map $f_{Z}^{t+1}: D_{t+1} \backslash Z \rightarrow R$ as follows.

(1) Suppose $Z \subseteq B \cap D_{t} . \exists$ sets $f_{Z}^{t+1}=f_{Z}^{t} \cup h_{Z}: D_{t+1} \backslash Z \rightarrow R$. This is well defined since for $x \in \operatorname{dom} f_{Z}^{t} \cap \operatorname{dom} h_{Z}=\left(D_{t} \backslash Z\right) \cap(B \backslash Z)$, we have $h_{Z}(x)=h_{Z}^{0}(x)=f_{Z}^{t}(x)$, and $\operatorname{dom} f_{Z}^{t+1}=\left(D_{t} \backslash Z\right) \cup(B \backslash Z)=D_{t+1} \backslash Z$, as required.

We remark here that we cannot guarantee that $f_{Z}^{t+1}$ is $1-1$. While $p$ is a 'new' node, $f_{Z}^{t+1}(p)$ may be a 'new' element of $R$ for some $Z$ and an 'old' one in $\operatorname{rng}\left(f_{Z}^{t}\right)$ for other $Z$. This is why we do not use strict networks $N_{X}^{\nu}$ in the definition of $\mathcal{S}_{n}(\mathcal{A})$ and why we do not require that $N_{X}^{\nu}(i, j) \leq 1^{\prime} \Rightarrow i \sim^{\nu} j$. See our discussion of loose representations earlier.

(2) Suppose $Z \subseteq D_{t}$ and $Z \nsubseteq B$. $\exists$ lets $f_{Z}^{t+1}: D_{t+1} \backslash Z \rightarrow R$ be an arbitrary extension of $f_{Z}^{t}: D_{t} \backslash Z \rightarrow R$ to the new node $p$.

(3) Suppose $Z \subseteq B$ and $Z \nsubseteq D_{t}$ (so $p \in Z$ ). $\exists$ lets $f_{Z}^{t+1}: D_{t+1} \backslash Z \rightarrow R$ be an arbitrary extension of $h_{Z}: B \backslash Z \rightarrow R\left(h_{Z} \in \mathcal{H}\right)$.

(4) Finally, if both $Z \nsubseteq D_{t}$ and $Z \nsubseteq B$, then $\exists$ chooses an arbitrary map $f_{Z}^{t+1}: D_{t+1} \backslash Z \rightarrow R$.

Let $\mathcal{F}_{t+1}=\left\{f_{Z}^{t+1}: Z \in\left[D_{t+1}\right]^{n-3}\right\}$. The pair $\left(D_{t+1}, \mathcal{F}_{t+1}\right)$ is $\exists$ 's response to $\forall$ 's move in round $t$. We check that it is satisfactory. Clearly, $D_{t+1} \supseteq D_{t}$ and $f_{Z}^{t+1} \supseteq f_{Z}^{t}$ for each $Z \in\left[D_{t}\right]^{n-3}$, so condition (4.3) for $t+1$ follows. Since $f_{Z}^{t+1} \supseteq h_{Z}$ for $Z \in[B]^{n-3}$, we have $\operatorname{Net}\left(D_{t+1}, \mathcal{F}_{t+1}\right)(\bar{b})=\operatorname{Net}(B, \mathcal{H})(\bar{b})=\nu^{\prime}$, as required.

Finally, let $\delta \leq \kappa$ be a limit ordinal, let $\left(D_{t}, \mathcal{F}_{t}\right)$ be the pair that was in play at the start of round $t$, for each $t<\delta$, and assume that (4.3) holds for these $t$. Define $D_{\delta}=\bigcup_{t<\delta} D_{t}$. By (4.3), $f_{Z}^{\delta}=\bigcup\left\{f_{Z}^{t}: t<\delta, Z \subseteq D_{t}\right\}$ is a well-defined map from $D_{\delta} \backslash Z$ to $R$ for each $Z \in\left[D_{\delta}\right]^{n-3}$. Define $\mathcal{F}_{\delta}=\left\{f_{Z}^{\delta}: Z \in\left[D_{\delta}\right]^{n-3}\right\}$. If $\delta<\kappa$, the pair at the start of round $\delta$ is now defined to be $\left(D_{\delta}, \mathcal{F}_{\delta}\right)$. (Clearly, (4.3) then holds for $t=\delta$.) For $\delta=\kappa,\left(D_{\delta}, \mathcal{F}_{\delta}\right)$ is the outcome of the game. 
Now consider a play of the game in which $\forall$ chooses $\nu \in \mathcal{S}$ (say) in the initial round and then plays, at some (possibly later) stage of the game, every move that ever becomes possible, and in which $\exists$ responds to $\forall$ 's moves as just described. Let $\left(D_{\kappa}, \mathcal{F}_{\kappa}\right)$ be the outcome of the game and put $M_{\nu}=$ $\operatorname{Net}\left(D_{\kappa}, \mathcal{F}_{\kappa}\right)$. We assume without loss of generality that the $M_{\nu}(\nu \in \mathcal{S})$ have pairwise disjoint domains. We now define $h: \mathcal{P}_{n}(\mathcal{A}) \rightarrow \wp\left(\bigcup_{\nu \in \mathcal{S}}{ }^{n} M_{\nu}\right)$ by $h(r)=\bigcup_{\nu \in \mathcal{S}}\left\{\bar{a} \in{ }^{n} M_{\nu}: M_{\nu}(\bar{a}) \in r\right\}$ for each $r \in \mathcal{P}_{n}(\mathcal{A})$. It can be checked that $h$ is a complete representation of $\mathcal{P}_{n}(\mathcal{A})$.

\section{Definition 4.8.}

(1) A signature $L$ is said to be ( $n$-dimensional) pseudo-diagonal-free if $\left\{+,-, 0,1, \mathrm{c}_{i}: i<n\right\} \subseteq L \subseteq\left\{+,-, 0,1, \mathrm{c}_{i}, \mathrm{~d}_{i j}, \mathrm{~s}_{\sigma}: i, j<n, \sigma: n \rightarrow n\right\}$.

(2) A pseudo-diagonal-free reduct of $\mathcal{P}_{n}(\mathcal{A})$ is a reduct of $\mathcal{P}_{n}(\mathcal{A})$ to an $n$ dimensional pseudo-diagonal-free signature.

(3) The cylindric reduct of $\mathcal{P}_{n}(\mathcal{A})$ is the reduct of $\mathcal{P}_{n}(\mathcal{A})$ to the signature $\left\{+,-, 0,1, \mathrm{c}_{i}, \mathrm{~d}_{i j}: i, j<n\right\}$ of $n$-dimensional cylindric algebras.

(4) Let $L$ be a pseudo-diagonal-free signature and $\mathcal{C}$ an $L$-algebra. The diagonal-free reduct of $\mathcal{C}$ is the reduct of $\mathcal{C}$ to $\left\{+,-, 0,1, c_{i}: i<n\right\}$, the signature of $n$-dimensional diagonal-free cylindric algebras.

\section{Proposition 4.9.}

(1) Suppose that the algebra $\mathcal{P}_{n}(\mathcal{A})$ is completely representable. Then every pseudo-diagonal-free reduct of $\mathcal{P}_{n}(\mathcal{A})$ is completely representable.

(2) Suppose that some pseudo-diagonal-free reduct of $\mathcal{P}_{n}(\mathcal{A})$ is completely representable. Then the diagonal-free reduct of $\mathcal{P}_{n}(\mathcal{A})$ is completely representable.

Proof. This is trivial.

The following proposition is a simple adaptation of Lemmas 1.4-1.7 and Theorem 1.8 of Johnson [12] to complete representations. Recall that an algebra $\mathcal{C}$ with signature $\left\{+,-, 0,1, \mathrm{c}_{i}, \mathrm{~d}_{i j}: i, j<n\right\}$ is an $n$-dimensional cylindric algebra if it satisfies the following axioms (from [4]), where $i, j, k<n$ and $x, y$ are arbitrary elements of $\mathcal{C}$ :

$\mathrm{C} 0$. the boolean reduct of $\mathcal{C}$ is a boolean algebra,

C1. $\mathrm{c}_{i} 0=0$,

C2. $x \leq \mathrm{c}_{i} x$,

C3. $\mathrm{c}_{i}\left(x \cdot \mathrm{c}_{i} y\right)=\mathrm{c}_{i} x \cdot \mathrm{c}_{i} y$,

C4. $\mathrm{c}_{i} \mathrm{c}_{j} x=\mathrm{c}_{j} \mathrm{c}_{i} x$,

C5. $\mathrm{d}_{i i}=1$,

C6. if $k \neq i, j$, then $\mathrm{d}_{i j}=\mathrm{c}_{k}\left(\mathrm{~d}_{i k} \cdot \mathrm{d}_{k j}\right)$,

C7. if $i \neq j$, then $\mathrm{c}_{i}\left(\mathrm{~d}_{i j} \cdot x\right) \cdot \mathrm{c}_{i}\left(\mathrm{~d}_{i j} \cdot-x\right)=0$.

Let $\mathcal{C}, \mathcal{C}^{\prime}$ be $n$-dimensional cylindric algebras. We say that $\mathcal{C}^{\prime}$ is a complete subalgebra of $\mathcal{C}$, and write $\mathcal{C}^{\prime} \subseteq{ }^{c} \mathcal{C}$, if $\mathcal{C}^{\prime}$ is a subalgebra of $\mathcal{C}$ and $\sum^{\mathcal{C}} X \in \mathcal{C}^{\prime}$ 
for each $X \subseteq \mathcal{C}^{\prime}$ such that $\sum^{\mathcal{C}} X$ exists. We say that $\mathcal{C}$ is completely generated by a subset $X \subseteq \mathcal{C}$ if whenever $X \subseteq \mathcal{C}^{\prime} \subseteq{ }^{c} \mathcal{C}$, we have $\mathcal{C}^{\prime}=\mathcal{C}$.

Proposition 4.10 (essentially Johnson). Let $\mathcal{C}$ be an $n$-dimensional cylindric algebra completely generated by $\{c \in \mathcal{C}: \Delta c \neq n\}$, where (recall) we define $\Delta c=\left\{i<n: c_{i} c \neq c\right\}$. Suppose that the diagonal-free reduct $\mathcal{D}$ (say) of $\mathcal{C}$ is completely representable. Then $\mathcal{C}$ is also completely representable.

Proof. First suppose that $\mathcal{D}$ is simple. Let $h: \mathcal{D} \rightarrow \mathcal{Q}$ be a complete representation, where $U_{i}(i<n)$ are sets, $P=\prod_{i<n} U_{i}$, and

$$
\mathcal{Q}=\left(\wp(P), \cup, \backslash, \emptyset, P, C_{i}^{P}: i<n\right),
$$

where $C_{i}^{P} X=\left\{\bar{a} \in P: \exists \bar{b} \in X\left(\bar{a}={ }_{i} \bar{b}\right)\right\}$ for $X \subseteq P$ and $i<n$, as in Definition 2.4.

Claim 1. We can assume that $U_{i}=U_{j}$ for each $i, j<n$, and if $\bar{a} \in P, i, j<n$, and $a_{i}=a_{j}$, then $\bar{a} \in h\left(\mathrm{~d}_{i j}\right)$.

Proof of claim. Let $\delta=\prod_{i, j<n} \mathrm{~d}_{i j} \in \mathcal{D}$. As $\mathcal{C}$ is a cylindric algebra, for each $i<n$ we have $\mathrm{c}_{0} \cdots \mathrm{c}_{i-1} \mathrm{c}_{i+1} \cdots \mathrm{c}_{n-1} \delta=1$. So for each $u \in U_{i}$, there is $\bar{a} \in h(\delta)$ with $a_{i}=u$. Hence we may choose a function $s_{i}: U_{i} \rightarrow h(\delta)$ such that $\left(s_{i}(u)\right)_{i}=u$ for each $u \in U_{i}$.

Now let $U$ be the disjoint union of the $U_{i}(i<n)$. Let $t_{i}: U \rightarrow U_{i}$ be the surjection given by $t_{i}(u)=\left(s_{j}(u)\right)_{i}$, where $u \in U_{j}$. Define

$$
\begin{aligned}
& g: \mathcal{D} \rightarrow\left(\wp\left({ }^{n} U\right), \cup, \backslash, \emptyset,{ }^{n} U, C_{i}^{U}: i<n\right) \quad \text { by } \\
& g: d \mapsto\left\{\bar{a} \in{ }^{n} U:\left(t_{0}\left(a_{0}\right), \ldots, t_{n-1}\left(a_{n-1}\right)\right) \in h(d)\right\} .
\end{aligned}
$$

Then (see [12, Lemma 1.4]) $g$ is a representation of $\mathcal{D}$. Moreover, since $h$ is complete, it is atomic (see Remark 2.5), and hence plainly, so is $g$. So $g$ is also a complete representation of $\mathcal{D}$. Finally, suppose that $\bar{a} \in{ }^{n} U$ satisfies $a_{i}=a_{j}$ with $a_{i} \in U_{k}$, say, where $k<n$. Let $\bar{b}=s_{k}\left(a_{i}\right)=s_{k}\left(a_{j}\right) \in h(\delta)$. Then $t_{i}\left(a_{i}\right)=b_{i}$ and $t_{j}\left(a_{j}\right)=b_{j}$. So $\left(t_{0}\left(a_{0}\right), \ldots, t_{n-1}\left(a_{n-1}\right)\right)$ agrees with $\bar{b}$ on coordinates $i, j$. Since $\bar{b} \in h(\delta) \subseteq h\left(\mathrm{~d}_{i j}\right)$ and $\Delta \mathrm{d}_{i j}=\{i, j\}$, we see that $\left(t_{0}\left(a_{0}\right), \ldots, t_{n-1}\left(a_{n-1}\right)\right) \in h\left(\mathbf{d}_{i j}\right)$, and so $\bar{a} \in g\left(\mathrm{~d}_{i j}\right)$, as required. This proves the claim.

From now on, we assume that the complete representation $h$ satisfies the properties of Claim 1. For distinct $i, j<n$ define $\sim_{i j}=\left\{\left(a_{i}, a_{j}\right): \bar{a} \in h\left(\mathrm{~d}_{i j}\right)\right\}$, a binary relation on $U$.

Claim 2. $\sim_{i j}=\sim_{01}$, and $\sim_{01}$ is an equivalence relation on $U$.

Proof of claim. This is [12, Lemma 1.6].

For $\bar{a}, \bar{b} \in{ }^{n} U$, define $\bar{a} \sim \bar{b}$ iff $a_{i} \sim 01 b_{i}$ for each $i<n$. Plainly, $\sim$ is an equivalence relation on ${ }^{n} U$. We let

$$
E=\{d \in \mathcal{D}: h(d) \text { is a union of } \sim \text {-classes }\} .
$$

Claim 3. $\{d \in \mathcal{D}: \Delta d \neq n\} \subseteq E$. 
Proof of claim. This is [12, Lemma 1.7].

Claim 4. $E$ is the domain of a complete subalgebra $\mathcal{E} \subseteq$ $\subseteq$.

Proof of claim. We have $\left\{0,1, \mathrm{~d}_{i j}: i, j<n\right\} \subseteq E$ by Claim 3 since $\Delta 0=\Delta 1=$ $\emptyset$ and $\Delta \mathrm{d}_{i j}=\{i, j\} \neq n$ (as $n \geq 3$ ). If $h(d)$ is a union of $\sim$-classes, then so is ${ }^{n} U \backslash h(d)=h(-d)$. If $S \subseteq E$ and $\sum^{\mathcal{D}} S$ exists, then as $h$ is a complete representation, $h\left(\sum^{\mathcal{D}} S\right)=\bigcup h[S]$, a union of $\sim$-classes - so $\sum^{\mathcal{D}} S \in E$ as well. Finally, if $e \in E$, then $\mathrm{c}_{i} e \in E$ by Claim 3 (as $i \notin \Delta\left(\mathrm{c}_{i} e\right)$ ). This proves the claim.

By the assumption in the proposition, $\mathcal{E}=\mathcal{C}$. Now define $V=U / \sim_{01}$, and

$$
\begin{aligned}
& g: \mathcal{C} \rightarrow\left(\wp\left({ }^{n} V\right), \cup, \backslash, \emptyset,{ }^{n} V, D_{i j}^{V}, C_{i}^{V}: i, j<n\right) \text { by } \\
& g: c \mapsto\left\{\left(a_{0} / \sim_{01}, \ldots, a_{n-1} / \sim_{01}\right): \bar{a} \in h(c)\right\}, \text { for } c \in \mathcal{C} .
\end{aligned}
$$

It can be checked that $g$ is an atomic, and hence complete, representation of C. In particular, $a_{i} / \sim_{01}=a_{j} / \sim_{01}$ iff $a_{i} \sim_{i j} a_{j}$ (by Claim 2) iff $\bar{a} \in h\left(\mathrm{~d}_{i j}\right.$ ) (since $\Delta \mathrm{d}_{i j}=\{i, j\}$ ).

Now we drop the assumption that $\mathcal{D}$ is simple. Suppose that $h: \mathcal{D} \rightarrow \mathcal{Q}=$ $\prod_{k \in K} \mathcal{Q}_{k}$ is a complete representation, as in Definition 2.4(2). Fix $k \in K$, let $\pi_{k}: \mathcal{Q} \rightarrow \mathcal{Q}_{k}$ be the canonical projection, and let $\mathcal{D}_{k}=\operatorname{rng}\left(\pi_{k} \circ h\right)$. We may define diagonal elements in $\mathcal{D}_{k}$ by $\mathrm{d}_{i j}=\pi_{k}\left(h\left(\mathrm{~d}_{i j}^{\mathcal{C}}\right)\right)$ : this expands $\mathcal{D}_{k}$ to a cylindric-type algebra $\mathcal{C}_{k}$ that is a homomorphic image of $\mathcal{C}$, and hence is a cylindric algebra with diagonal-free reduct $\mathcal{D}_{k}$. The inclusion map $\iota_{k}: \mathcal{D}_{k} \rightarrow$ $\mathcal{Q}_{k}$ is a complete representation of $\mathcal{D}_{k}$. Also, since plainly

$$
\pi_{k}[h[\{c \in \mathcal{C}: \Delta c \neq n\}]] \subseteq\left\{c \in \mathcal{C}_{k}: \Delta c \neq n\right\},
$$

and $\pi_{k}, h$ preserve arbitrary sums, we see that $\mathcal{C}_{k}$ is completely generated by $\left\{c \in \mathcal{C}_{k}: \Delta c \neq n\right\}$. Now $c_{0} \cdots c_{n-1} x$ is a discriminator term in $\mathcal{Q}_{k}$, and it follows that $\mathcal{D}_{k}$ is simple. So by the above, $\mathcal{C}_{k}$ has a complete representation $g_{k}: \mathcal{C}_{k} \rightarrow \mathcal{Q}_{k}^{\prime}$, say. Choose such a $g_{k}$ and $\mathcal{Q}_{k}^{\prime}$ for each $k$. Then it is not hard to see that $g: \mathcal{C} \rightarrow \prod_{k \in K} \mathcal{Q}_{k}^{\prime}$ given by $g(c)_{k}=g_{k}\left(\pi_{k}(h(c))\right)$ is a complete representation of $\mathcal{C}$.

Corollary 4.11. Suppose that the diagonal-free reduct $\mathcal{D}\left(\right.$ say) of $\mathcal{P}_{n}(\mathcal{A})$ is completely representable. Then the cylindric reduct $\mathcal{C}$ of $\mathcal{P}_{n}(\mathcal{A})$ is also completely representable.

Proof. First we check that $\mathcal{C}$ is a cylindric algebra. $\mathcal{C}$ satisfies $\mathrm{C} 0-\mathrm{C} 4$ since $\mathcal{D}$ is representable. Any $\nu \in \mathcal{S}_{n}(\mathcal{A})$ satisfies $i \sim^{\nu} i$, so $\nu \in \mathrm{d}_{i i}$. Hence, C5 holds. For C6, by Lemma 3.6, $\mathrm{c}_{k}\left(\mathrm{~d}_{i k} \cdot \mathrm{d}_{k j}\right)=\mathrm{s}_{[k / i]} \mathrm{d}_{k j}=\left\{\nu: \nu^{[k / i]} \in \mathrm{d}_{k j}\right\}=$ $\left\{\nu: k\left(\sim^{\nu}\right)_{[k / i]} j\right\}=\left\{\nu: i \sim^{\nu} j\right\}=\mathrm{d}_{i j}$. For C7, again by Lemma 3.6,

$$
\begin{aligned}
\mathrm{c}_{i}\left(\mathrm{~d}_{i j} \cdot x\right) \cdot \mathrm{c}_{i}\left(\mathrm{~d}_{i j} \cdot-x\right) & =\mathrm{s}_{[i / j]} x \cdot \mathrm{s}_{[i / j]}(-x) \\
& =\left\{\nu: \nu^{[i / j]} \in x\right\} \cap\left\{\nu: \nu^{[i / j]} \in-x\right\}=\emptyset .
\end{aligned}
$$

We now show that each atom $\nu$ of $\mathcal{C}$ is in the subalgebra generated by $\{c \in \mathcal{C}: \Delta c \neq n\}$. In fact, we have $\nu=\prod_{i<n} \mathrm{c}_{i} \nu$. To prove this, we take 
an arbitrary atom $\nu^{\prime} \leq \prod_{i<n} c_{i} \nu$, so that $\nu \equiv_{i} \nu^{\prime}$ for every $i<n$, and prove that $\nu^{\prime}=\nu$. Certainly, $\sim^{\nu}=\sim^{\nu^{\prime}}$ because $n \geq 3$ and $\sim^{\nu}={ }_{i} \sim^{\nu^{\prime}}$ for every $i<n$. Write $\sim$ for $\sim^{\nu}$ and let $X \in H(\sim)$; we check that $N_{X}^{\nu}=N_{X}^{\nu^{\prime}}$. Take any $i, j \in X$ and representatives $k_{1}, \ldots, k_{n-3}$ of the $\sim$-classes in $n \backslash X$. Let $l \in n \backslash\left\{i, j, k_{1}, \ldots, k_{n-3}\right\}$. Then $(n \backslash(X \cup\{l\})) / \sim=(n \backslash X) / \sim$, which yields $|(n \backslash(X \cup\{l\})) / \sim|=n-3$. So $X$ and $X$ match for $\sim$ off $l$, and since $\nu \equiv_{l} \nu^{\prime}$, we have $N_{X}^{\nu}={ }_{l} N_{X}^{\nu^{\prime}}$. Hence, $N_{X}^{\nu}(i, j)=N_{X}^{\nu^{\prime}}(i, j)$. As $i, j \in X$ were arbitrary, this shows that $N_{X}^{\nu}=N_{X}^{\nu^{\prime}}$. So $\nu=\nu^{\prime}$, as desired.

Since every element of $\mathcal{C}$ is a sum of atoms, $\mathcal{C}$ is completely generated by $\{c \in \mathcal{C}: \Delta c \neq n\}$. The corollary now follows from Proposition 4.10.

Proposition 4.12. Suppose that the cylindric reduct $\mathcal{C}\left(\right.$ say) of $\mathcal{P}_{n}(\mathcal{A})$ is completely representable. Then $\mathcal{A}$ is completely representable as well.

Proof. We can find $\delta=\left(\sim^{\delta}, N_{X}^{\delta}: X \in H(\sim)\right) \in \mathcal{S}=\mathcal{S}_{n}(\mathcal{A})$ with $i \not{ }^{\delta} j$ for all $i<j<n$ : i.e., $\sim^{\delta}$ is equality on $n$. Since $\mathcal{C}$ is completely representable, there is a complete homomorphism $h: \mathcal{C} \rightarrow\left(\wp\left({ }^{n} M\right), \cup, \backslash, \emptyset, M, C_{i}^{M}, D_{i j}^{M}: i, j<n\right)$ for some set $M$, such that $h(\delta) \neq \emptyset$. Define $M(\bar{a})=\prod\{c \in \mathcal{C}: \bar{a} \in h(c)\}$ for $\bar{a} \in{ }^{n} M$; by Remark 2.5, this is an atom of $\mathcal{C}$ and we identify it with the corresponding element of $\mathcal{S}$ as usual. By Lemma 3.6, the substitutions $\mathrm{s}_{[i / j]}$ $(i, j<n)$ are term-definable, so are respected by $h$. So for each $i, j<n$ and $\bar{a} \in{ }^{n} M$, we have

M1. $a_{i}=a_{j}$ iff $M(\bar{a}) \in \mathrm{d}_{i j}$, iff $i \sim^{M(\bar{a})} j$,

M2. $M(\bar{a} \circ[i / j])=M(\bar{a})^{[i / j]}$.

Take $\bar{d} \in{ }^{n} M$ satisfying $M(\bar{d})=\delta$. For each $i<j<n$, we have $i \chi^{\delta} j$, so by M1, $d_{i} \neq d_{j}$. Consequently, $|M| \geq|\operatorname{rng} \bar{d}|=n$ and $Z=\left\{d_{3}, \ldots, d_{n-1}\right\} \in$ $[M]^{n-3}$. Put

$$
\begin{aligned}
\bar{z} & =\left(d_{3}, \ldots, d_{n-1}\right), \\
R & =M \backslash Z \neq \emptyset .
\end{aligned}
$$

Plainly, if $p, q, r \in R$ and $M(p, q, r, \bar{z})=\nu$, say, then $3 \in H\left(\sim^{\nu}\right)$, so $N_{3}^{\nu}$ is defined. We now define an atomic $\mathcal{A}$-network over $R$ as follows. For $p, q \in R$, we define

$$
R(p, q)=N_{3}^{\nu}(0,1) \in \operatorname{At} \mathcal{A}, \text { where } \nu=M(p, q, q, \bar{z}) .
$$

Claim. If $a_{0}, a_{1}, a_{2} \in R, i, j<3, a_{i}=p, a_{j}=q$, and $\nu=M\left(a_{0}, a_{1}, a_{2}, \bar{z}\right)$, then $R(p, q)=N_{3}^{\nu}(i, j)$.

Proof of claim. Choose $k \in 3 \backslash\{0, i\}$ and let $\sigma=[k / j] \circ[0 / i] \circ[1 / k] \circ[2 / 1]$. Then $\left(a_{0}, a_{1}, a_{2}, \bar{z}\right) \circ \sigma=\left(a_{i}, a_{j}, a_{j}, \bar{z}\right)=(p, q, q, \bar{z})$, so by $\mathrm{M} 2, M(p, q, q, \bar{z})=\nu^{\sigma}$. Вy definition, $R(p, q)=N_{3}^{\nu^{\sigma}}(0,1)=N_{\sigma_{\nu}(3)}^{\nu}(\sigma(0), \sigma(1))=N_{3}^{\nu}(i, j)$, as claimed.

We will establish in a standard way the following properties. They will show that $R$ is a network and yields a complete representation of $\mathcal{A}$.

(1) $R(p, p) \leq 1$ ' for each $p \in R$. Let $M(p, p, p, \bar{z})=\nu$, say. By M1, $0 \sim^{\nu} 1$. So $R(p, p)=N_{3}^{\nu}(0,1) \leq 1$. 
(2) $R(q, p)=R(p, q)^{\smile}$ for each $p, q \in R$. Let $M(p, q, q, \bar{z})=\nu$. By the claim, $R(q, p)=N_{3}^{\nu}(1,0)=N_{3}^{\nu}(0,1)^{\smile}=R(p, q)^{\smile}$.

(3) $R(p, q) \leq R(p, r) ; R(r, q)$ for each $p, q, r \in R$. Let $\nu=M(p, q, r, \bar{z})$ and $N=N_{3}^{\nu}$. By the claim and because $N$ is a network, $R(p, q)=N(0,1) \leq$ $N(0,2) ; N(2,1)=R(p, r) ; R(r, q)$.

(4) If $p, q \in R, a, b \in$ At $\mathcal{A}$, and $R(p, q) \leq a$; b, then there is $r \in R$ with $R(p, r)=a$ and $R(r, q)=b$. To see this, using that $|R| \geq 3$, choose $r^{\prime} \in R \backslash\{p, q\}$ and let $\nu=M\left(p, q, r^{\prime}, \bar{z}\right)$, so by the claim, $R(p, q)=$ $N_{3}^{\nu}(0,1) \leq a ; b$. By properties of relation algebras, there is an atomic $\mathcal{A}$-network $N$ with domain 3 and such that $N={ }_{2} N_{3}^{\nu}, N(0,2)=a$, and $N(2,1)=b$. Let $\nu^{\prime}$ be identical to $\nu$ except that $N_{3}^{\nu^{\prime}}=N$. It can be verified that $\nu^{\prime} \in \mathcal{S}$ and $\nu^{\prime} \equiv_{2} \nu$. Hence, $\nu \leq \mathrm{c}_{2} \nu^{\prime}$. As $h$ preserves $\mathrm{c}_{2}$, there is $r \in M$ with $M(p, q, r, \bar{z})=\nu^{\prime}$. As $2 \not \nu^{\prime} j$ for each $j \in n \backslash 3$, we have $r \in R$. By the claim, $R(p, r)=N_{3}^{\nu^{\prime}}(0,2)=N(0,2)=a$ and $R(r, q)=N_{3}^{\nu^{\prime}}(2,1)=N(2,1)=b$.

Now define a congruence $\approx$ on $R$ by $p \approx q$ iff $R(p, q) \leq 1$ '. Let $[p]$ denote the congruence class of $p \in R$. Since $\mathcal{A}$ is simple, it is standard to check that the map $h: \mathcal{A} \rightarrow \mathfrak{R e}(R / \approx)$ given by $h(x)=\{([p],[q]): p, q \in R, R(p, q) \leq x\}$ (for $x \in \mathcal{A})$ is a complete representation of $\mathcal{A}$.

Theorem 4.13. Let $\mathcal{A}$ be a simple atomic relation algebra and let $L$ be an $n$-dimensional pseudo-diagonal-free signature. Then $\mathcal{A}$ is completely representable iff the $L$-reduct of $\mathcal{P}_{n}(\mathcal{A})$ is completely representable.

Proof. This follows from Propositions 4.7, 4.9, 4.12, and Corollary 4.11.

\section{Undecidability of representability}

We can now obtain our first main results.

Corollary 5.1. For each finite $n \geq 3$ and each signature $L$ satisfying

$$
\left\{+,-, 0,1, \mathrm{c}_{i}: i<n\right\} \subseteq L \subseteq\left\{+,-, 0,1, \mathrm{c}_{i}, \mathrm{~d}_{i j}, \mathrm{~s}_{\sigma}: i, j<n, \sigma: n \rightarrow n\right\},
$$

it is undecidable whether a finite L-algebra is representable as an algebra of $n$-ary relations.

Proof. Suppose for contradiction that there is an algorithm A to determine whether a finite $L$-algebra is representable. We can now decide effectively whether a finite simple relation algebra $\mathcal{A}$ is representable by constructing the (finite) $L$-reduct $\mathcal{C}$ of $\mathcal{P}_{n}(\mathcal{A})$ and returning the answer $\mathrm{A}(\mathcal{C})$. By Theorem 4.13 , this is the correct answer since for finite algebras, every representation is complete.

However, by [8, Theorem 18.13(1)], it is undecidable whether a finite simple relation algebra is representable. This is a contradiction, and so no such algorithm A can exist. 
Theorem 5.2. For finite $n \geq 3$, it is undecidable whether a finite algebra of the following kinds is representable:

(1) n-dimensional cylindric algebra,

(2) n-dimensional polyadic algebra,

(3) n-dimensional polyadic equality algebra,

(4) n-dimensional diagonal-free cylindric algebra (a known result [10]).

Proof. Take the case of cylindric algebras. Let $\mathrm{C}$ be the class of all $n$-dimensional cylindric-type algebras; $\left(\mathrm{RCA}_{n}\right.$ and) $\mathrm{CA}_{n}$ denote the classes of (representable) $n$-dimensional cylindric algebras. By Corollary 5.1, there is no algorithm to decide whether a finite member of $C$ is in $\mathrm{RCA}_{n}$. Since $\mathrm{RCA}_{n} \subseteq$ $\mathrm{CA}_{n} \subseteq \mathrm{C}$ and it is decidable whether a finite member of $\mathrm{C}$ is in $\mathrm{CA}_{n}$, it follows by elementary recursion theory that there is no algorithm to decide whether a finite $n$-dimensional cylindric algebra is in $\mathrm{RCA}_{n}$. The other cases are proved similarly.

Remark 5.3. Examination of the proof shows that Theorem 5.2 also holds for simple algebras, since if $\mathcal{A}$ is simple, then so is every pseudo-diagonal-free reduct of $\mathcal{P}_{n}(\mathcal{A})$. (This can be proved by showing that $\mathrm{c}_{0} \cdots \mathrm{c}_{n-1} \mathrm{c}_{n-1} \cdots \mathrm{c}_{0} x$ is a discriminator term.) It also holds for algebras generated by $<n$-dimensional elements (see Corollary 4.11).

It trivially follows that the classes $\mathrm{RCA}_{n}, \mathrm{RPA}_{n}, \mathrm{RPEA}_{n}, \mathrm{RDf}_{n}$ of representable algebras of the kinds in Theorem 5.2 are not finitely axiomatisable in first-order, second-order, or $k$ th-order logic (any finite $k$ ), in first-order logic extended by fixed point operators, etc., since a finite axiomatisation in a logic where evaluation in finite structures is effective would immediately yield an algorithm to decide representability.

\section{Complete representations}

It was shown in [6] that for any ordinal $\alpha \geq 3$, the classes of completely representable relation algebras and completely representable $\alpha$-dimensional cylindric algebras are non-elementary. Here, we extend this result to more classes of algebra. Essentially the same result can be found in [13], although an analogue of Proposition 4.10 appears to be missing.

To begin, let $X$ be an infinite set and write $\wp_{\omega}(X)$ for the set of countable subsets of $X$. A club in $\wp_{\omega}(X)$ is a subset $C \subseteq \wp_{\omega}(X)$ with the following properties:

closed: if $C_{0} \subseteq C_{1} \subseteq \cdots$ and $C_{i} \in \mathrm{C}$ for each $i<\omega$, then $\bigcup_{i<\omega} C_{i} \in \mathrm{C}$, unbounded: for each $Y \in \wp_{\omega}(X)$, there is $C \in \mathrm{C}$ containing $Y$.

Now fix a non-principal ultrafilter $D$ on $\omega$. For a structure $M$, we write $M^{*}$ for the ultrapower $M^{\omega} / D$. By $[6, \S 3.4, \S 3.6]$, there is an (integral and hence) simple atomic relation algebra $\mathcal{A}$ such that:

(1) $\mathcal{A}$ is not completely representable, 
(2) the set $\mathrm{C}$ of countable elementary subalgebras of $\mathcal{A}^{*}$ that are completely representable forms a club in $\wp_{\omega}\left(\mathcal{A}^{*}\right)$.

It follows easily that the class of completely representable relation algebras is not elementary. But with our earlier results, these properties also imply the same for pseudo-diagonal-free algebras, as we now show. The relation algebra $\mathcal{A}$ remains as above. Note that by Loś's theorem [1, Theorem 4.1.9], $\mathcal{A}^{*}$ and $\mathcal{P}_{n}(\mathcal{A})^{*}$ are atomic algebras.

Lemma 6.1. $\mathcal{P}_{n}(\mathcal{A})^{*}$ is isomorphic to a dense subalgebra of $\mathcal{P}_{n}\left(\mathcal{A}^{*}\right)$.

Proof. By a theorem of Goldblatt [2, Lemma 3.6.5], there is an algebra embedding $\theta:\left(\mathcal{S}_{n}(\mathcal{A})^{+}\right)^{*} \rightarrow\left(\mathcal{S}_{n}(\mathcal{A})^{*}\right)^{+}$given by

$\theta\left(\left(r_{i}: i<\omega\right) / D\right)=\left\{\bar{x} / D: \bar{x} \in{ }^{\omega} \mathcal{S}_{n}(\mathcal{A}),\left\{i<\omega: x_{i} \in r_{i}\right\} \in D\right\} \quad\left(\subseteq \mathcal{S}_{n}(\mathcal{A})^{*}\right)$

for $r_{i} \subseteq \mathcal{S}_{n}(\mathcal{A})(i<\omega)$. It can be verified that $\mathcal{S}_{n}(-)$ commutes with ultraproducts (e.g., because $\mathcal{S}_{n}(\mathcal{A})$ is first-order interpretable in $\mathcal{A}$ ). So $\mathcal{S}_{n}(\mathcal{A})^{*} \cong$ $\mathcal{S}_{n}\left(\mathcal{A}^{*}\right)$. Together this yields an embedding $\theta^{\prime}:\left(\mathcal{S}_{n}(\mathcal{A})^{+}\right)^{*} \rightarrow \mathcal{S}_{n}\left(\mathcal{A}^{*}\right)^{+}$, that is, $\theta^{\prime}: \mathcal{P}_{n}(\mathcal{A})^{*} \rightarrow \mathcal{P}_{n}\left(\mathcal{A}^{*}\right)$. It can be checked that $\theta^{\prime}$ maps $\operatorname{At} \mathcal{P}_{n}(\mathcal{A})^{*}$ surjectively onto $\operatorname{At} \mathcal{P}_{n}\left(\mathcal{A}^{*}\right)$. Since $\mathcal{P}_{n}\left(\mathcal{A}^{*}\right)$ is atomic, the $\theta^{\prime}$-image of $\mathcal{P}_{n}(\mathcal{A})^{*}$ is a dense subalgebra of $\mathcal{P}_{n}\left(\mathcal{A}^{*}\right)$.

From now on, we shall identify $\mathcal{P}_{n}(\mathcal{A})^{*}$ with this dense subalgebra, and thereby assume that $\mathcal{P}_{n}(\mathcal{A})^{*} \subseteq \mathcal{P}_{n}\left(\mathcal{A}^{*}\right)$, the two having the same set of atoms (i.e., $\mathcal{S}_{n}\left(\mathcal{A}^{*}\right)$, up to isomorphism). We can now obtain the main result of this section. In the proof, we assume familiarity with basic model theory of elementary substructures and chains: see, e.g., [1].

Theorem 6.2. Let $n \geq 3$ be finite, and let $L$ be an $n$-dimensional pseudodiagonal-free signature. The class of completely representable L-algebras is not elementary.

Proof. As above, let $\mathrm{C}$ be the club of completely representable countable elementary subalgebras of $\mathcal{A}^{*}$. They are all atomic. We will define countable elementary chains of algebras:

$$
\begin{aligned}
& \overbrace{\mathcal{B}_{0} \subseteq \mathcal{B}_{1} \subseteq \cdots}^{\text {in C }} \preceq \mathcal{A}^{*}, \\
& \mathcal{D}_{0} \preceq \mathcal{D}_{1} \preceq \ldots \preceq \mathcal{P}_{n}(\mathcal{A})^{*} \subseteq \text { dense } \mathcal{P}_{n}\left(\mathcal{A}^{*}\right) .
\end{aligned}
$$

We let $\mathcal{B}_{0} \in \mathrm{C}$ be arbitrary and $\mathcal{D}_{0} \preceq \mathcal{P}_{n}(\mathcal{A})^{*}$ any countable elementary subalgebra. Given $l<\omega$ such that $\mathcal{B}_{l}, \mathcal{D}_{l}$ are defined, we define $\mathcal{B}_{l+1}, \mathcal{D}_{l+1}$ as follows:

(1) Clearly, $\mathcal{S}_{n}\left(\mathcal{B}_{l}\right)$ is a countable subset of $\mathcal{S}_{n}\left(\mathcal{A}^{*}\right) \subseteq \mathcal{P}_{n}(\mathcal{A})^{*}$. Using the downward Löwenheim-Skolem-Tarski theorem, let $\mathcal{D}_{l+1}$ be any countable elementary subalgebra of $\mathcal{P}_{n}(\mathcal{A})^{*}$ containing $\mathcal{S}_{n}\left(\mathcal{B}_{l}\right)$ and $\mathcal{D}_{l}$. 
(2) Because $\mathcal{D}_{l+1} \preceq \mathcal{P}_{n}(\mathcal{A})^{*}$, we see that $\mathcal{D}_{l+1}$ is atomic. Choose $\mathcal{B}_{l+1} \in \mathrm{C}$ containing $\mathcal{B}_{l}$ and such that $\operatorname{At} \mathcal{D}_{l+1} \subseteq \mathcal{S}_{n}\left(\mathcal{B}_{l+1}\right)$. This is possible because $\mathrm{C}$ is a club and only countably many atoms of $\mathcal{A}^{*}$ are involved in atomic $\mathcal{A}^{*}$-networks occurring in atoms of $\mathcal{D}_{l+1}$.

This completes the definition of the $\mathcal{B}_{l}$ and $\mathcal{D}_{l}$. Let $\mathcal{B}_{\omega}=\bigcup_{l<\omega} \mathcal{B}_{l} \subseteq \mathcal{A}^{*}$ and $\mathcal{D}_{\omega}=\bigcup_{l<\omega} \mathcal{D}_{l} \preceq \mathcal{P}_{n}(\mathcal{A})^{*}$. As $\mathrm{C}$ is a club, $\mathcal{B}_{\omega} \in \mathrm{C}$, so $\mathcal{B}_{\omega}$ is a completely representable relation algebra. By Theorem $4.13, \mathcal{P}_{n}\left(\mathcal{B}_{\omega}\right)$ is a completely representable polyadic equality algebra. For each $l<\omega$, we arranged that $\mathcal{D}_{l}$ is atomic and

$$
\mathcal{S}_{n}\left(\mathcal{B}_{l}\right) \subseteq \text { At } \mathcal{D}_{l+1} \subseteq \mathcal{S}_{n}\left(\mathcal{B}_{l+1}\right)
$$

From this and properties of elementary chains, we see that $\mathcal{D}_{\omega}$ is atomic and

$$
\operatorname{At} \mathcal{D}_{\omega}=\bigcup_{l<\omega} \operatorname{At} \mathcal{D}_{l}=\bigcup_{l<\omega} \mathcal{S}_{n}\left(\mathcal{B}_{l}\right)=\mathcal{S}_{n}\left(\mathcal{B}_{\omega}\right)
$$

and it follows that $\mathcal{D}_{\omega}$ is a dense subalgebra of $\mathcal{P}_{n}\left(\mathcal{B}_{\omega}\right)$. By Remark 2.5, $\mathcal{D}_{\omega}$ is completely representable. Clearly its $L$-reduct is a completely representable $L$-algebra.

But $\mathcal{D}_{\omega}$ is elementarily equivalent to $\mathcal{P}_{n}(\mathcal{A})^{*}$, and hence also to $\mathcal{P}_{n}(\mathcal{A})$, and this obviously remains true for the $L$-reducts of these algebras. As $\mathcal{A}$ is not completely representable, by Theorem 4.13 , neither is the $L$-reduct of $\mathcal{P}_{n}(\mathcal{A})$. So the class of completely representable $L$-algebras is not closed under elementary equivalence and cannot be elementary.

Corollary 6.3. For each finite $n \geq 3$, the completely representable $n$-dimensional polyadic algebras, polyadic equality algebras, cylindric algebras, and diagonal-free cylindric algebras do not form elementary classes.

Remark 6.4. For infinite dimensions, the corollary is true for all pseudodiagonal-free signatures containing the diagonal elements. This can be shown directly by a simple cardinality argument [6, Corollary 26]. It also holds for infinite-dimensional diagonal-free cylindric algebras. To see this, using the proof of Theorem 6.2, take two elementarily equivalent 3-dimensional diagonalfree-type algebras $\mathcal{B}, \mathcal{C}$, where $\mathcal{B}$ is completely representable and $\mathcal{C}$ is not. Let $\alpha \geq 3$ be any ordinal. Expand $\mathcal{B}, \mathcal{C}$ to $\alpha$-dimensional diagonal-free-type algebras $\mathcal{B}_{\alpha}, \mathcal{C}_{\alpha}$ by defining $\mathrm{c}_{i} x=x$ for all $x$ and all $3 \leq i<\alpha$. Plainly, $\mathcal{B}_{\alpha}$ is elementarily equivalent to $\mathcal{C}_{\alpha}$, and it can be checked that $\mathcal{B}_{\alpha}$ is completely representable and $\mathcal{C}_{\alpha}$ is not.

The case of infinite-dimensional polyadic algebras (without diagonals) is not covered by this argument. We do not know whether the corollary holds for them, or (even in finite dimensions) for numerous other kinds of algebras, including Pinter's substitution algebras [24] and various relativised set algebras. 


\section{Conclusion}

Here we very briefly list some possible further work. We already suggested some in Remark 6.4. We could also ask for necessary and sufficient conditions on a simple atomic relation algebra $\mathcal{A}$ for the cylindric reduct of $\mathcal{P}_{n}(\mathcal{A})$ to be in classes such as $\mathrm{CA}_{n}, \mathbf{S} \mathfrak{N r}_{n} \mathrm{CA}_{n+m}$, etc. On the other side, what properties of $\mathcal{P}_{n}(\mathcal{A})$ (if any) flow from $\mathcal{A} \in \mathrm{RA}_{k}, \mathcal{A} \in \mathrm{S} \mathfrak{R a C A}_{k}$, etc, for $k \geq 5$ ?

It could be interesting to use the construction of Section 3 , or a stronger one, to lift other relation algebra results to cylindric algebras and polyadic algebras. An example is the problem of whether, for finite $n \geq 3$, the class of polyadic (equality) atom structures whose complex algebras are representable is elementary. In the notation of [3, p. 556], this is asking whether the classes StrRPA and StrRPEA ${ }_{n}$ are elementary. $[9,14]$ prove that $\operatorname{StrRCA}_{n}$ and $\operatorname{StrRDf}_{n}$, respectively, are non-elementary for every finite $n \geq 3$. This problem may perhaps be solved by adapting the known proofs for relation algebras or cylindric algebras, but a direct reduction from the relation algebra case [8, Chapter 14] may be an alternative.

It may also be interesting (and necessary for the above) to extend the construction of Section 3 to arbitrary relation algebras that are not necessarily atomic. It seems that this can be done, but it is complicated.

It may also be worth giving a representability-preserving reduction from $\mathrm{CA}_{n}$ to $\mathrm{CA}_{n+1}$, and similarly for polyadic (equality) algebras.

Acknowledgements. Thanks to Robin Hirsch and Agi Kurucz for several very helpful suggestions, and to the referee for a magnificent detailed report with more than eight pages of discussion and several very helpful suggestions.

\section{REFERENCES}

[1] Chang, C.C., Keisler, H.J.: Model Theory, 3rd edn. North-Holland, Amsterdam (1990)

[2] Goldblatt, R.: Varieties of complex algebras. Ann. Pure Appl. Logic 44, 173-242 (1989)

[3] Goldblatt, R.: Elementary generation and canonicity for varieties of boolean algebras with operators. Algebra Universalis 34, 551-607 (1995)

[4] Henkin, L., Monk, J.D., Tarski, A.: Cylindric Algebras Part I. North-Holland (1971)

[5] Henkin, L., Monk, J.D., Tarski, A.: Cylindric Algebras Part II. North-Holland (1985)

[6] Hirsch, R., Hodkinson, I.: Complete representations in algebraic logic. J. Symbolic Logic 62, 816-847 (1997)

[7] Hirsch, R., Hodkinson, I.: Representability is not decidable for finite relation algebras. Trans. Amer. Math. Soc. 353, 1403-1425 (2001)

[8] Hirsch, R., Hodkinson, I.: Relation Algebras by Games. Studies in Logic and the Foundations of Mathematics, vol. 147. North-Holland, Amsterdam (2002)

[9] Hirsch, R., Hodkinson, I.: Strongly representable atom structures of cylindric algebras. J. Symbolic Logic 74, 811-828 (2009)

[10] Hirsch, R., Hodkinson, I., Kurucz, A.: On modal logics between $K \times K \times K$ and $S 5 \times S 5 \times S 5$. J. Symbolic Logic 67, 221-234 (2002)

[11] Hodkinson, I., Wolter, F., Zakharyaschev, M.: Decidable and undecidable fragments of first-order branching temporal logics. In: Proc. 17th IEEE Symposium on Logic in Computer Science (LICS), pp. 393-402. IEEE Inc. (2002) 
[12] Johnson, J.S.: Nonfinitizability of classes of representable polyadic algebras. J. Symbolic Logic 34, 344-352 (1969)

[13] Khaled, M., Sayed Ahmed, T.: On complete representations of algebras of logic. Logic J. IGPL 17, 267-272 (2009)

[14] Kurucz, A.: On the complexity of modal axiomatisations over many-dimensional structures. In: Beklemishev, L., Goranko, V., Shehtman, V. (eds.) Advances in Modal Logic, vol. 8, pp. 256-270. College Publications (2010)

[15] Lyndon, R.: The representation of relational algebras. Ann. Math. 51, 707-729 (1950)

[16] Maddux, R.D.: Topics in Relation Algebra. PhD thesis, University of California, Berkeley (1978)

[17] Maddux, R.D.: Non-finite axiomatizability results for cylindric and relation algebras. J. Symbolic Logic 54, 951-974 (1989)

[18] Maddux, R.D.: Introductory course on relation algebras, finite-dimensional cylindric algebras, and their interconnections. In: Andréka, H., Monk, J.D., Németi, I. (eds.) Algebraic Logic. Colloq. Math. Soc. J. Bolyai, vol. 54, pp. 361-392. North-Holland, Amsterdam (1991)

[19] Maddux, R.D.: Relation Algebras. Studies in Logic and the Foundations of Mathematics, vol. 150. Elsevier, Amsterdam (2006)

[20] Monk, J.D.: Studies in Cylindric Algebra. PhD thesis, University of California, Berkeley (1961)

[21] Monk, J.D.: On representable relation algebras. Michigan Math. J. 11, 207-210 (1964)

[22] Monk, J.D.: Nonfinitizability of classes of representable cylindric algebras. J. Symbolic Logic 34, 331-343 (1969)

[23] Németi, I.: Free Algebras and Decidability in Algebraic Logic. Doctoral Dissertation with the Academy, Budapest (1986)

[24] Pinter, C.: Cylindric algebras and algebras of substitutions. Trans. Amer. Math. Soc. 175, 167-179 (1973)

[25] Sági, G.: A note on algebras of substitutions. Studia Logica 72, 265-284 (2002)

[26] Tarski, A.: On the calculus of relations. J. Symbolic Logic 6, 73-89 (1941)

\section{IAN HODKINSON}

Department of Computing, Imperial College London, SW7 2AZ, UK

e-mail: i.hodkinson@imperial.ac.uk

URL: www.doc.ic.ac.uk/ imh/

Open Access This article is distributed under the terms of the Creative Commons Attribution License which permits any use, distribution, and reproduction in any medium, provided the original author(s) and the source are credited. 